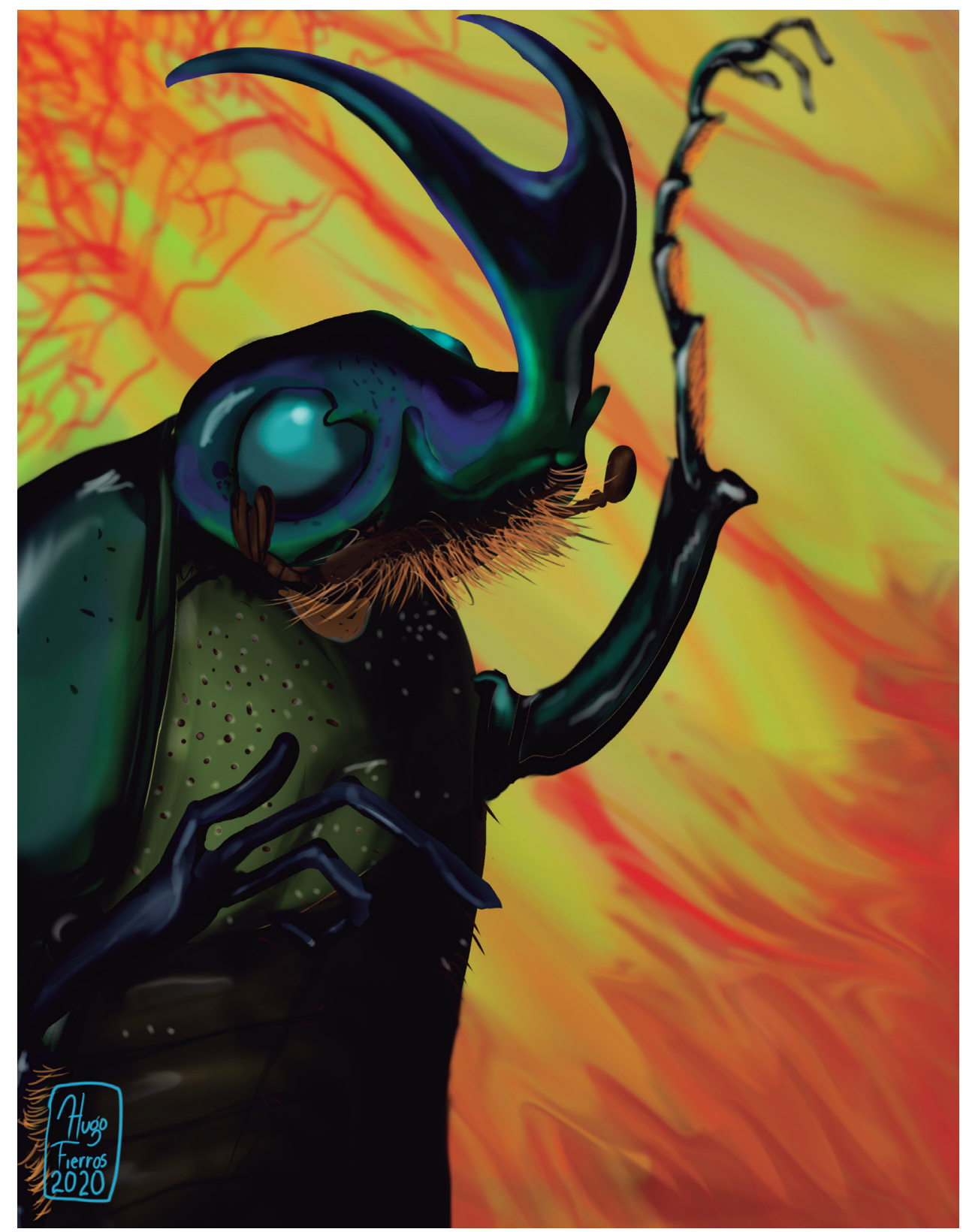

Dugesiana, Año 28, No. 1, (enero-junio, primer semestre de 2021), es una publicación semestral, editada por la Universidad de Guadalajara, a través del Centro de Estudios en Zoología, por el Centro Universitario de Ciencias Biológicas y Agropecuarias. Camino Ramón Padilla Sánchez \# 2100, Nextipac, Zapopan, Jalisco, Tel. 37771150 ext. 33218, http://148.202.248.171/dugesiana/index.php/DUG/index, glenusmx@gmail.com. Editor responsable: José Luis Navarrete-Heredia. Reserva de Derechos al Uso Exclusivo 04-2009-062310115100-203, ISSN: $2007-$ 9133, otorgados por el Instituto Nacional del Derecho de Autor. Responsable de la última actualización de este número: José Luis Navarrete-Heredia, Editor y Ana Laura González-Hernández, Asistente Editorial. Fecha de la última modificación 15 defebrero 2021, con un tiraje de un ejemplar.

Las opiniones expresadas por los autores no necesariamente reflejan la postura del editor de la publicación.

Queda estrictamente prohibida la reproducción total o parcial de los contenidos e imágenes de la publicación sin previa autorización de la Universidad de Guadalajara. 


\section{Distribución vertical y estacional de coleópteros (Coleoptera) en un bosque artificial de un área de conservación afectada por la urbanización en el centro de México}

\section{Vertical and seasonal distribution of beetles (Coleoptera) in an artificial forest of a conservation area affected by urbanization in the central of Mexico \\ Gabriela Medina Reyes ${ }^{1}$, Esteban Jiménez-Sánchez ${ }^{1 *}$, Roberto Quezada-García ${ }^{2}$, Santiago \\ Zaragoza-Caballero $^{3}$ y Jorge R. Padilla-Ramírez ${ }^{1}$}

${ }^{1}$ Laboratorio de Zoología, Facultad de Estudios Superiores Iztacala, Universidad Nacional Autónoma de México. Av. de los Barrios 1, Los Reyes Iztacala, 54090, Tlalnepantla, Estado de México, México; ${ }^{2}$ Centre d'Étude de la Forêt (CEF) y Département des Sciences du Bois et de la Forêt, Faculté de Foresterie, Géographie et Géomatique, Université Laval, Canadá; ${ }^{3}$ Colección Nacional de Insectos, Departamento de Zoología, Instituto de Biología, Universidad Nacional Autónoma de México, Apartado postal 70-153, 04510 Ciudad de México, México. *Autor de correspondencia: estjimsan@gmail.com

'gabidelcorral@gmail.com, ORCID: https://orcid.org/0000-0002-2564-2515

1,*estjimsan@gmail.com, ORCID: https://orcid.org/0000-0001-7310-7747

2roberto_queza71@hotmail.com, ORCID: https://orcid.org/0000-0003-1448-8847

${ }^{3}$ zaragoza@ib.unam.mx, ORCID: https://orcid.org/0000-0002-0235-318X

1jorgepr@unam.mx, ORCID: https://orcid.org/0000-0002-6368-8397

\section{RESUMEN}

Se estudió la diversidad vertical y estacional de los coleópteros en un bosque artificial en el centro de México. Los muestreos se realizaron mensualmente durante un año, usando trampas de intercepción de vuelo colocadas a diferentes alturas (1 m y $5 \mathrm{~m})$ y trampas pitfall $(0 \mathrm{~m})$. Se obtuvo un total de 456 individuos agrupados en 31 familias y 126 morfoespecies. Se registró mayor riqueza y abundancia a $1 \mathrm{~m}$, y los valores inferiores a nivel del suelo. La diversidad fue significativamente mayor en el dosel a $5 \mathrm{~m}$ que en los estratos inferiores. La fauna difirió considerablemente entre estratos. Por ejemplo, el recambio espacial en la composición de especies fue el responsable de la diversidad beta total. Así mismo, más del 70\% de las especies estuvieron representadas por uno o dos individuos y fueron exclusivas de algún estrato. La abundancia y la riqueza de especies disminuyeron durante el periodo de sequía, con excepción del nivel del suelo. Los taxones indicadores de cada estrato fueron determinados usando el valor indicador (IndVal) (Carabidae indicador a 0 m; Chrysomelidae, Melolonthinae y Latriididae a $1 \mathrm{~m}$; Bruchinae y Aleocharinae a $5 \mathrm{~m}$ ). Los fungívoros, depredadores y saprófagos agruparon a 25 familias; las restantes seis fueron herbívoras. Los distintos gremios tróficos también mostraron una estratificación y en algunos casos su distribución vertical varió con la época del año. De manera general, la riqueza de morfoespecies y familias fue más parecida a la observada en bosques templados; sin embargo, los estimadores de especies y muestreos realizados con otros métodos de recolecta, sugieren que debe existir una mayor riqueza a pesar de la perturbación. La estratificación observada para los ensambles de coleópteros, puede ser explicada por la distribución espacial de los recursos alimentarios y su variación temporal relacionada con las condiciones de estacionalidad climática.

Palabras clave: trampa intercepción de vuelo, trampa de caída, Estado de México, Sierra de Guadalupe, matorral xerófilo.

\footnotetext{
ABSTRACT

Vertical and seasonal diversity of beetles (coleoptera) were studied in an artificial forest in central Mexico. Monthly samples were collected for a year, with flight intercept traps set at different heights (1 and $5 \mathrm{~m})$, and pitfall traps at ground level (0 m). A total of 456 individuals of 31 families and 126 morphospecies were collected. The highest richness and abundance were observed at 1 $\mathrm{m}$, and the lower values were at ground level. Beetle diversity was higher in the canopy $(5 \mathrm{~m})$ than it was in lower strata. The fauna differed considerably between strata. For example, the spatial turnover in species composition was responsible for most of the beta diversity. Indeed, more than $70 \%$ of collected species were represented by one or two individuals which exhibited preference for a given stratum. Drought decreased abundance and species richness except at ground level. The indicator taxa for each stratum were determined using the indicator value (IndVal) (Carabidae indicator at $0 \mathrm{~m}$; Chrysomelidae, Melolonthinae y Latriididae at $1 \mathrm{~m}$; Bruchinae and Aleocharinae at $5 \mathrm{~m}$ ). The fungivores, predators and saprophagous belonged to 25 families the remaining six were herbivores. The different trophic guilds also showed a stratification, and, in some cases, its vertical distribution changed with the season of the year. In general, the richness of morphospecies and families was more similar to that observed in the temperate forest. However, the estimators and samplings carried out with others collection methods suggest that a higher richness could exist in spite of anthropic disturbances. The stratification obtained for the beetle assemblages can be explained by the spatial distribution of food resources. The temporal variation is in turn related with climatic seasonality.
}

Key words: flight intercep trap, pitfall trap, Estado de México, Sierra de Guadalupe, xeric shrub. 
Son numerosos los estudios sobre distribución vertical de la diversidad de artrópodos e insectos en zonas templadas y tropicales y, en éstos, siempre se registra al orden Coleoptera como uno de los grupos más numerosos y diversos (Basset et al. 1992; Kato et al. 1995; Hill y Cermak 1997; Basset 1988; Preisser et al. 1998; Basset et al. 2001; $\mathrm{Su}$ y Woods 2001). Lo cual se ve reflejado en el alto número de investigaciones que utilizan a los coleópteros como objeto de estudio de la distribución vertical en los bosques (Wagner 2000; Chung 2004; Stork y Grimbacher 2006; Grimbacher y Stork 2007; Ulyshen y Hanula 2007; Schroeder et al. 2009; Ulyshen et al. 2010; Davis et al. 2011; Maguire et al. 2014; Kuprin y Drumont 2016; Vegabadillo et al. 2018; Hernández-Jáuregui et al. 2019).

Se ha observado que los ensambles de insectos del dosel son diferentes de los que habitan más cerca del suelo (Hill y Cermak 1997; Preisser et al. 1998); este patrón de estratificación ocurre también para la fauna de coleópteros (Chung 2004; Stork y Grimbacher 2006; Ulyshen y Hanula 2007; Kuprin y Drumont 2016). Aunque es controversial si un estrato contribuye más a la diversidad que otro, varios estudios sugieren que la contribución del dosel y la del sotobosque son muy similares (Stork y Grimbacher 2006; Ulyshen y Hanula 2007; Vega-badillo et al. 2018; Maguire et al. 2014), por lo que, es importante considerar los diferentes estratos en los programas de muestreo, para tener una mejor representación de la fauna de un sitio. La evidencia de una especialización al dosel y una mayor preferencia por el estrato, sugiere que el dosel debe ser considerado en la conservación como parte del manejo integral de los ecosistemas forestales (Davis et al. 2011).

Los coleópteros representan más de un tercio $(392,415$ especies descritas) de todas las especies de insectos (1,070,781 especies descritas) conocidas (Zhang 2013); aunado a su gran diversidad, su alimentación puede incluir recursos variados, tales como: follaje, flores, frutos, resinas, madera, hongos, carroña, excremento, presas y hospederos. Además, requieren diferentes hábitats distribuidos desde el suelo hasta la copa de los árboles. La disponibilidad de los recursos alimentarios es una de las determinantes más importantes en la distribución vertical de los artrópodos, además de múltiples factores, tales como el tiempo, estructura del bosque, composición de la comunidad de plantas, gradientes climáticos e interacciones interespecíficas, todo esto actuando simultáneamente (Ulyshen 2011).

En particular, el área donde se realizó la presente investigación, es un conjunto de montañas independientes que se encuentra en medio de la urbanización, la cual asciende sus laderas afectando la cobertura vegetal; si bien, la influencia antrópica ha ocurrido desde hace más de 2,000 años con una intensa deforestación (Lugo-Hubp y SalinasMontes 1996), la afectación ha sido muy acelerada en los últimos 30 años (Peña 2018). Por lo tanto, buena parte de la vegetación nativa (matorral xerófilo) ha desaparecido y ha sido sustituida por la introducción de especies arbóreas exóticas; creando bosques artificiales con una estructura diferente -con un dosel muy abierto de mayor altura que la que alcanzan las especies nativas, y aunado a la predominancia de vegetación secundaria anual, resultado de la gran perturbación del sitio (GCDMX 2016). En este sentido, los bosques secundarios tienen una menor diversidad de hábitats y recursos que los bosques primarios y soportan menor diversidad de artrópodos (Ulyshen 2011).

Por otro lado, la parte central de México, se caracteriza por presentar un periodo de sequía y uno de lluvias bien marcados, lo que provoca que la vegetación anual desaparezca y que varios árboles y arbustos pierdan sus hojas durante el periodo estival; lo cual tienen importantes implicaciones en la distribución estacional de los coleópteros, como se ha observado en algunos bosques urbanos de la zona de Guadalajara, Jalisco (NavarreteHeredia et al. 2012) o en las zonas agrícolas, urbanas y suburbanas de la región semiárida de la porción noroeste del Estado de México (Jiménez-Sánchez et al. 2019). Estos cambios estacionales también son muy característicos de los bosques caducifolios donde se ha observado que tienen importantes implicaciones en la distribución vertical de los artrópodos (Ulyshen y Hanula 2007; Ulyshen 2011; Vegabadillo et al. 2018).

En el presente estudio se plantean las siguientes preguntas para un bosque artificial en el centro de México: 1. ¿La riqueza, abundancia y composición de los ensambles de coleópteros es diferente entre los estratos de vegetación? 2. ¿Existe una variación temporal de los ensambles de coleópteros? 3. ¿Hay variación vertical y temporal de los gremios tróficos de coleópteros? Se espera en general, que la marcada estacionalidad climática y los cambios de la fenología y estructura de la vegetación a lo largo del año que esto conlleva, influyan en la distribución espacial vertical y temporal de los ensambles de coleópteros en los diferentes estratos. De acuerdo con Wardhaugh (2014), uno de los factores más importantes que influyen en este tipo de patrones entre los ensambles de insectos arbóreos, es la disponibilidad de recursos, que a su vez están relacionados con las condiciones abióticas, las cuales están cambiando continuamente a través del tiempo sobre todo en sistemas que experimentan una marcada estacionalidad climática.

La información generada, servirá como referencia para conocer cómo se ve alterada la diversidad del sitio por la perturbación de la vegetación original y aportar información para ayudar en los esfuerzos de manejo y conservación del área.

\section{MATERIAL Y MÉTODOS}

Área de estudio. Se ubicó en la Sierra de Guadalupe, específicamente en el "Centro Ecoturístico y de Educación Ambiental Sierra de Guadalupe" en Coacalco, Estado de México; dicha Sierra es un Área Natural Protegida localizada al norte de la Ciudad de México y los límites del Estado de México donde se ubica el $82 \%$ de su superficie (Cedillo et al. 2007) (Figura 1-I). La mancha urbana rodea 
y asciende las laderas montañosas, afectando su vegetación. En el periodo 1990-2017 el suelo urbano creció $22.8 \%$, con pérdida de una superficie neta de $25 \%$ de cobertura vegetal arbórea y pastizal, en tan solo 27 años (Peña 2018).

La Sierra de Guadalupe pertenece a la provincia fisiográfica Faja Volcánica Transmexicana. La altitud varia de la cota de 2,240 a los 2,800-3,000 msnm, y es un pequeño conjunto de elevaciones volcánicas independientes y sobrepuestas (Lugo-Hubp y Salinas-Montes 1996). El clima es templado subhúmedo con lluvias en verano $\mathrm{Cb}(\mathrm{w} 0)(\mathrm{w})$ (i')g; presenta temperatura media anual de $16.7^{\circ} \mathrm{C}$; enero es el mes más frío con una media de $13.1^{\circ} \mathrm{C}$ y junio el más cálido con $18.8^{\circ} \mathrm{C}$ (Cedillo et al. 2007). Con precipitación media anual de $733.9 \mathrm{~mm}$, enero es el mes más seco con 30 mm y junio el más húmedo con 152 mm (GCDMX 2016). El periodo de sequía va de noviembre a abril y el de lluvias de mayo a octubre (Peña 2018).

Los tipos de vegetación presentes en la Sierra de Guadalupe son el pastizal inducido, el matorral xerófilo y los bosques artificiales; con menor cobertura se encuentran los bosques naturales de encino y las nopaleras, de tal manera que la vegetación primaria se ha reducido sensiblemente dando lugar a la formación de asociaciones vegetales derivadas de las perturbaciones y la introducción de especies exóticas, producto de la reforestación con fines de restauración y conservación (Cedillo et al. 2007; GCDMX 2016). Esto último, es el caso particular del sitio donde se realizaron los muestreos, que se ubicó en un bosque artificial compuesto por plantaciones de Eucalyptus camaldulensis Dehnh. (eucalipto), Casuarina equisetifolia L. (casuarina), Cupressus lindleyi Klotzsch ex Endl. (cedro blanco), C. sempervirens L. (ciprés panteonero) y diversas especies de pinos, entre las que se encuentran Pinus cembroides Zucc., P. montezumae Lamb., P. patula Schltdl. and Cham. y P. radiata D.Don.; los cuales forman el estrato del dosel forestal superior con alturas que van de $5 \mathrm{~m}$ en los árboles más jóvenes a más de $10 \mathrm{~m}$, y dominado por eucaliptos, con sus copas muy separadas unas de otras (Figura 1-II). El estrato de sotobosque está dominado por plantas herbáceas anuales de Poaceae, Asteraceae, Fabaceae y Convolvulaceae, con escasos elementos de la vegetación nativa como Cactaceae: Opuntia sp. (nopales), Mammillaria sp. (biznaga), Asparagaceae: Agave sp. (maguey) y Fabaceae: Vachellia farnesiana (L.) Wight \& Arn. (huizache).

Muestreo. Se seleccionaron tres árboles de Eucalyptus camaldulensis que fue la especie más común, con una altura promedio de $10 \mathrm{~m}$, y ramas adecuadas para colgar las trampas, a lo largo de un transecto sobre la ladera norte de la Sierra (árbol 1: 19³6'27.0" N, 9905'35.0" $\mathrm{O}, 2,454 \mathrm{msnm}$; árbol 2: $19^{\circ} 36^{\prime} 24.6^{\prime \prime} \mathrm{N}, 9^{\circ} 05^{\prime} 33.5^{\prime} \mathrm{O}$, 2,471 msnm, árbol 3: 19³6’25.9'” N, 9905’30.2” O, 2,502 msnm) (Figura 1-I), con una separación aproximada de 100 metros entre éstos y una diferencia altitudinal promedio de 25 metros entre los sitios.

En cada árbol se colocaron tres trampas, dos de intercepción de vuelo, la primera de éstas a una altura promedio de $5 \mathrm{~m}$ en el dosel, en las ramas más bajas de las copas; la segunda se suspendió debajo del dosel a 1 $\mathrm{m}$ de altura y la tercera correspondió a una trampa pitfall colocada a nivel del suelo $(0 \mathrm{~m})$, todas ellas una debajo de la otra. En total se instalaron tres trampas por cada altura, con un total de nueve. Las trampas estuvieron activas siete días cada mes, tres días antes y tres días después de la fase de luna nueva, de agosto de 2017 a julio de 2018 .

Las trampas de intercepción de vuelo consistieron en dos tiras de PET transparente $(40 \mathrm{~cm} \times 60 \mathrm{~cm}$ ) cortadas por la mitad y ensambladas entre ellas para formar una barrera en forma de cruz de 20 x $30 \mathrm{~cm}$ de cada lado; y por debajo un cono del mismo material con un diámetro de $45 \mathrm{~cm}$ y $20 \mathrm{~cm}$ de profundidad, que en la parte inferior llevaba un bote de plástico de 1.51 de capacidad, $10 \mathrm{~cm}$ de diámetro y $17 \mathrm{~cm}$ de profundidad. En la parte superior de la trampa se colocó un círculo de plástico de $45 \mathrm{~cm}$ de diámetro, con un soporte para colgarla. La trampa pitfall consistió en un bote de plástico de 21 de capacidad, $18 \mathrm{~cm}$ de diámetro y $13 \mathrm{~cm}$ de profundidad que fue enterrado a ras del suelo, cubierto por una tapa de plástico de $27 \mathrm{~cm}$ de diámetro, colocada a $5 \mathrm{~cm}$ del borde superior del bote, para permitir la entrada de los organismos y a su vez evitar inundación por la lluvia. Como líquido conservador se utilizó en ambos tipos de trampa, una mezcla de sal con agua (50 g por litro) y el material obtenido fue colocado en alcohol al 70\% para su traslado al laboratorio.

Procesamiento del material entomológico. El material entomológico se separó e identificó hasta el nivel taxonómico posible. El 97\% de las especies que correspondió a 122 morfoespecies, fueron determinadas solamente a nivel de género, subfamilia o familia de Coleoptera, y solamente cuatro a nivel de especie. Se utilizó la clasificación de Bouchard et al. (2011) para organizar las familias. Para la identificación de los especímenes se utilizaron las claves de: Arnett y Thomas (2001), Arnett et al. (2002), NavarreteHeredia et al. (2002), Triplehorn y Johnson (2005) y por comparación con ejemplares de la Colección de Artrópodos de la Facultad de Estudios Superiores Iztacala (CAFESI), UNAM, donde además se depositaron los especímenes de referencia; así como, en la Colección Nacional de Insectos (CNIN) del Instituto de Biología, UNAM.

Análisis de datos. Se cuantificó el número de individuos (abundancia) y el número de morfoespecies (riqueza) para cada sitio, mes y trampa. Para evaluar las diferencias entre los componentes de la diversidad alfa y la eficiencia del muestreo en los sitios, se empleó el método propuesto por Chao et al. (2014), quienes utilizan los números de Hill, los cuales integran la riqueza de especies y su abundancia, donde ${ }^{0} \mathrm{D}$ es la riqueza de especies y conforme $q$ se incrementa, el valor de diversidad es influenciado más fuertemente por la abundancia de la especie más dominante. Por lo tanto, si todas las especies de la comunidad tienen exactamente la misma abundancia, entonces ${ }^{0} \mathrm{D},{ }^{1} \mathrm{D}$ y ${ }^{2} \mathrm{D}$ tendrán el mismo valor. Cuando la abundancia está distribuida menos 
uniformemente entre las especies, ${ }^{1} \mathrm{D}$ tendrá un menor valor que ${ }^{0} \mathrm{D}, \mathrm{y}{ }^{2} \mathrm{D}$ un valor inferior que ${ }^{1} \mathrm{D}$. Es decir que cuando la abundancia de una comunidad es menos uniforme, los ordenes más altos de diversidad tendrán valores inferiores que los de la riqueza de especies (Moreno et al. 2018). Para representar estas tendencias de la diversidad, para cada sitio se elaboraron las curvas integradas de extrapolación y rarefacción para los números de Hill (riqueza de especies (q $=0)$, diversidad de Shannon $(\mathrm{q}=1)$, concentración inversa de Simpson $(\mathrm{q}=2)$ ), con un intervalo de confianza de $95 \%$, con base en el método de Bootstrap (nboots $=50$ ); para ello se extrapoló duplicando el número de individuos de cada sitio.

Para comparar los valores de diversidad entre los sitios, se hicieron las curvas de extrapolación y rarefacción basados en el tamaño de la muestra para cada valor fijo de q, con un intervalo de confianza de $95 \%$, previamente se calculó el tamaño de muestra base. Se realizó un análisis de covarianza para observar las diferencias entre las curvas para cada grupo $(\mathrm{q}=0, \mathrm{q}=1$ y q $=2)$. Para saber qué tan completo fue el muestro para cada sitio, se construyó una curva de completitud de la muestra; posteriormente, se graficaron las curvas de extrapolación y rarefacción basados en la cobertura de la muestra para la diversidad. Los análisis se realizaron con la paquetería iNEXT 2.0.19 (Hsieh et al., 2016).

Para analizar la disimilitud entre los sitios y meses, se utilizó el método propuesto por Baselga (2010), que desglosa la diversidad beta $\left(\beta_{\mathrm{SOR}}\right)$ en sus componentes aditivos: i) el recambio espacial en la composición de especies $\left(\beta_{\mathrm{SIM}}\right)$, que corresponde al reemplazo de algunas especies por otras como consecuencia de variaciones ambientales, o limitaciones espaciales o históricas; y ii) el anidamiento de los ensambles de especies $\left(\beta_{\mathrm{NES}}\right)$, el cual ocurre cuando las biotas de sitios con menor número de especies son un subconjunto de sitios con una biota más rica. Estos análisis fueron realizados con la paquetería betapart de R (Baselga, 2010).

Para determinar las familias indicadoras de cada uno de los estratos, se realizó el método propuesto por Dufrêne y Legendre (1997). Este método consiste en evaluar la afinidad de un grupo a un sitio determinado, basado en la combinación de la abundancia de las especies con su ocurrencia en las trampas instaladas en cada uno de los estratos. Este método permite distinguir las especies que tienen una mayor afinidad a los diferentes hábitats mediante una técnica de ordenamiento jerárquica y no jerárquica de las ocurrencias. Este análisis se realizó, tomando en cuenta el año, el mes y el método de recolecta con la finalidad de no perder la variabilidad de las familias en los estratos; la significancia se observó a través de una prueba de Montecarlo con 999 permutaciones. Este análisis se realizó mediante la paquetería indicspecies (De Cáceres y Legendre 2009). Todos los análisis fueron llevados a cabo con el programa estadístico R Development Core Team, versión. 3.5.2. (2019).
En la comparación de la composición de especies y la diversidad beta entre los estratos, se hicieron dos análisis, uno incluyendo todas las especies y en otro se excluyeron las especies raras (singletons y doubletons), considerando que éstas últimas contribuyen poco a las medidas importantes de la función de los ecosistemas como son la biomasa, productividad o retención de nutrientes (Shwartz et al. 2000) y de esta manera comparar el patrón mostrado por las especies que estuvieron bien representadas en la muestra y que podrían ser consideradas como residentes. Así mismo, en el cuadro 5 solo se presentan los datos de captura de las familias con más de diez individuos, por ser las que muestran una frecuencia de captura mayor entre trampas y estratos. Por otra parte, los gremios tróficos (o posibles gremios tróficos) para cada familia en el cuadro 1, están basados en Stork y Grimbacher (1973), Thayer (2005) y Davis et al. (2011).

\section{RESULTADOS}

Abundancia y riqueza. Se obtuvieron un total de 456 coleópteros, agrupados en 31 familias y 126 morfoespecies. Las familias Curculionidae, Scarabaeidae y Staphylinidae fueron divididas en diez subfamilias debido a que, a diferencia del resto de las familias, éstas incluyen subfamilias cuyas especies tienen hábitos alimentarios y hábitats muy variados, que son importantes desde el punto de vista funcional o taxonómico; tal es el caso de Staphylinidae, la cual incluye algunas subfamilias exclusivamente depredadoras (Paederinae, Scydmaeninae y Staphylininae) o principalmente saprófagas (Oxytelinae), hasta subfamilias que puede agrupar especies de varios gremios (Aleocharinae); otro caso es la familia Scarabaeidae que tuvo subfamilias cuyos adultos son exclusivamente herbívoros (Cetoniinae y Melolonthinae) o saprófagos (Aphodiinae); mientras que dentro de Curculionidae la subfamilia Scolytinae es xilófaga/fungívora a diferencia del resto de los curculiónidos que son principalmente herbívoros (Cuadro 1).

Se registró mayor riqueza y abundancia en el sotobosque a $1 \mathrm{~m}$, seguido del dosel a $5 \mathrm{~m}$ y el suelo a $0 \mathrm{~m}$ (Cuadro 1). El $58 \%$ de las especies estuvieron representadas por un individuo (singletons) y $14.3 \%$ por dos individuos (doubletons). Entre $72 \%$ y $79 \%$ de las especies estuvieron representadas por singletons y doubletons en todos los estratos (Cuadro 2), lo que indica una alta proporción de especies raras.

Completitud de la muestra y diversidad. Los valores de cobertura de la muestra más altos se obtuvieron a $1 \mathrm{~m}$ y $0 \mathrm{~m}$, con $81 \%$ y $80 \%$ respectivamente, el valor más bajo a $5 \mathrm{~m}(68 \%)$; en todos los casos los valores indican que el muestreo en los tres estratos es incompleto (Figura 2). $\mathrm{La}$ diversidad del orden $\mathrm{q}=0$ (riqueza de especies) fue más alta en el sotobosque a $1 \mathrm{~m}$ (87) y el dosel a $5 \mathrm{~m}$ (55) con diferencias significativas $(\mathrm{p}=0.0001)$ entre ambos estratos; la menor diversidad fue en el suelo a $0 \mathrm{~m}$ (19) con diferencias significativas $(\mathrm{p}=0.0001)$ con respecto al 
dosel, pero sin diferencias $(\mathrm{p}=0.09)$ con el sotobosque. Mientras que la diversidad de los órdenes $\mathrm{q}=1 \mathrm{y} q=2$, fue significativamente mayor $(p=0.0001)$ en el dosel que en el sotobosque y el suelo; entre estos dos últimos no hubo diferencias significativas $(\mathrm{p}=0.09)$ (Figura 3$)$. Esto obedece a que las especies del dosel tuvieron una distribución más equitativa de la abundancia: solo dos especies tuvieron más de diez individuos y representaron el 19\% de la abundancia total; mientras que, en los estratos a $0 \mathrm{~m}$, dos especies agruparon el $38 \%$ y a $1 \mathrm{~m}$ una sola especie representó el $36 \%$ de la abundancia total.

Composición de especies. La composición de la fauna difirió considerablemente entre los diferentes estratos; entre $1 \mathrm{~m}$ y $5 \mathrm{~m}$ compartieron el mayor número de morfoespecies con 19 (cuatro de Bruchinae, Zygogramma sp., Scolytinae sp., dos de Curculionidae, Photinus sp. 2, Melandrydae sp. 1, Mordellidae sp. 3, Nitidulidae sp. 1, Ptilidae sp. 1, Ptinidae sp. 1, M. mexicanus, dos de Aleocharinae, Hymenorus sp. 1 y Statira sp.) (15\% del total de especies) incluidas en 11 familias; a su vez estos dos niveles compartieron con el nivel del suelo otras seis morfoespecies pertenecientes a seis familias (Anthicidae sp. 1, Carabidae sp., Chrysomelidae sp. 1, Cymatodera sp. 2, Monotomidae sp. y Aleocharinae sp. 7) (4.7\%); cinco morfoespecies (Latridiidae sp. 1, Amecocerus sp., Mycetophagidae sp. 1, Anotylus sp. 1 y Platystethus sp. 1) (3.9\%) representantes de cuatro familias estuvieron en todos los estratos, y las restantes 96 morfoespecies (76.2\%) fueron exclusivas de algún estrato (Cuadro 1). De las especies no compartidas, $61.4 \%$ (47\% del total de especies) fueron exclusivas del estrato de $1 \mathrm{~m}, 30.2 \%$ (23\%) del dosel a $5 \mathrm{~m}$ y $8.3 \%(6.3 \%)$ del suelo $(0 \mathrm{~m})$.

Cuando se excluyen las especies raras (singletons y doubletons), los estratos de $1 \mathrm{~m}$ y $5 \mathrm{~m}$ compartieron 15 morfoespecies ( $43 \%$ del total); a su vez estos dos niveles compartieron cinco morfoespecies (14.3\%) con el suelo; cinco $(14.2 \%)$ estuvieron en todos los estratos, y las restantes diez (28.6\%) fueron exclusivas del algún estrato. De las especies no compartidas, $70 \%$ (20\% del total de especies) fueron exclusivas del estrato de $1 \mathrm{~m}, 20 \%(5.7 \%$ del total de especies) del dosel a $5 \mathrm{~m}$ y $10 \%$ ( $2.8 \%$ del total de especies) del suelo $(0 \mathrm{~m})$.

Diversidad beta entre estratos. Al comparar la disimilitud entre los estratos, se obtuvo el valor de diversidad beta total de $\beta_{\mathrm{SOR}}=0.77 \mathrm{y}$ el desglose de sus componentes. El recambio espacial en la composición de especies $\left(\beta_{\mathrm{SIM}}=0.6\right)$ y el anidamiento $\left(\beta_{\mathrm{NES}}=0.17\right)$ indican que el primero de éstos es el principal responsable del valor de la diversidad beta total. Cuando se eliminan las especies raras el patrón se mantiene y la diversidad beta total se eleva $\left(\beta_{\mathrm{SOR}}=0.9, \beta_{\mathrm{SIM}}=0.75, \beta_{\mathrm{NES}}=0.15\right)$.

Estacionalidad. La abundancia y la riqueza disminuyeron en todos los estratos en el periodo de sequía, con excepción del nivel del suelo donde este patrón no fue tan marcado (Figura 4). En todos los estratos, más de la mitad de las especies (56-69\%) fueron exclusivas del periodo de lluvias, seguido por la sequía (20-25\%), un porcentaje menor de especies (10-18\%) estuvieron presentes en ambos periodos; a nivel de familia se observó un patrón similar, aunque destaca un porcentaje alto de familias presentes en ambos periodos (entre $25 \%$ y $39 \%$ ). La abundancia en todos los estratos fue mayor durante las lluvias (Cuadro 3$)$. El valor de diversidad beta total $\left(\beta_{\text {SOR }}\right.$ $=0.7)$ para comparar la disimilitud entre épocas sugieren que el recambio en la composición de especies $\left(\beta_{\mathrm{SIM}}=0.54\right.$; $\left.\beta_{\mathrm{NES}}=0.16\right)$ es el responsable de la diferencia entre éstas, es decir que las variaciones en la precipitación anual podrían estar influyendo en la presencia de algunas especies.

Variación de familias y especies entre estratos. Los resultados muestran una variación entre los estratos con respecto a la distribución de las familias; solamente siete de las 31 familias estuvieron presentes en los tres estratos, ocho familias se compartieron entre los estratos a $1 \mathrm{~m}$ y $5 \mathrm{~m}$; a su vez estas dos alturas compartieron cuatro familias con el nivel del suelo (estrato a $0 \mathrm{~m}$ ). El suelo no tuvo ninguna familia exclusiva, mientras que la altura de $1 \mathrm{~m}$ presentó el mayor número de familias exclusivas (ocho familias), seguido por el dosel (cuatro familias) (Cuadro 1). El mayor número de familias indicadoras de acuerdo con los valores de IndVal se registró a $1 \mathrm{~m}$ con seis familias; mientras que, a $0 \mathrm{~m}$ y $5 \mathrm{~m}$ solo tuvieron dos (Cuadro 4).

El número de morfoespecies entre las familias y subfamilia osciló entre 1 y 12 . Al considerar únicamente a las 15 que tuvieron más de cuatro morfoespecies en total, Tenebrionidae fue la de mayor riqueza a nivel del suelo (Cuadro 1). En el sotobosque (1 m) fueron: Cantharidae, Chrysomelidae, Bruchinae (Chrysomelidae), Coccinellidae, Mordellidae, Ptinidae, Melolonthinae (Scarabaeidae) y Alleculinae (Tenebrionidae). Mientras que, en el dosel $(5 \mathrm{~m})$ fueron: Carabidae, Scolytinae (Curculionidae) y Aleocharinae (Staphylinidae) (Cuadro 1).

El número de individuos entre las familias y subfamilias tuvo un rango de 1 a 121, de las cuales 15 tuvieron más de diez organismos en total; de éstas, Carabidae y Tenebrionidae predominaron en el suelo. En el sotobosque fueron: Cantharidae, Chrysomelidae, Latridiidae, Melyridae, Cetoniinae (Scarabaeidae), Melolonthinae (Scarabaeidae), Oxytelinae (Staphylinidae) y Alleculinae (Tenebrionidae). En el dosel fueron: Bruchinae (Chrysomelidae), Scolytinae (Curculionidae) y Aleocharinae (Staphylinidae) (Cuadro 1). En el caso de la especie de cetonino Euphoria basalis (Gory y Percheron, 1833), fue exclusiva del sotobosque, mientras que los melolontinos estuvieron representados por tres especies, entre éstas, Macrodactylus mexicanus Burmeister, 1845, que fue la más abundante a $1 \mathrm{~m}$ (106 individuos), ésta y otra morfoespecie también se presentaron en el dosel (5 $\mathrm{m})$.

Solamente diez de las 31 familias registradas estuvieron representadas por más de diez individuos, las cuales agruparon 380 individuos $(83.3 \%$ del total) y 84 morfoespecies (66.6\%). Entre esas familias también se encuentran seis de las siete familias que fueron registradas 
en todos los estratos, las cuales mantienen su afinidad principalmente por los estratos superiores (Cuadro 5).

Composición de gremios tróficos. La mayoría de las familias son consideradas principalmente de hábitos alimentarios fungívoros (9 familias), depredadores (8) y saprófagos (8); los gremios con menor representación fueron los herbívoros (6) y los xilófagos (2). Todos los gremios tróficos tuvieron mayor riqueza de familias entre 1 m y $5 \mathrm{~m}$ del suelo; las dos familias xilófagas, Cerambycidae y Scolytinae (Curculionidae), solo se registraron en estos dos últimos estratos (Cuadro 1, Figura 5).

Con respecto a la variación estacional y vertical, las familias saprófagas se concentraron a nivel del suelo durante la sequía y al final de las lluvias, el resto de los meses estuvieron en los estratos superiores. Los herbívoros estuvieron exclusivamente en los estratos superiores durante todo el año con excepción de septiembre, cuando Chrysomelidae y Curculionidae también se registraron en el suelo; también se observó un incremento en el número de familias herbívoras durante las lluvias, principalmente en el estrato de $1 \mathrm{~m}$ (Figura 5).

Los depredadores se registraron en todos los estratos durante la mayor parte del año, aunque con un mayor número de familias en las lluvias y en los estratos superiores (Figura 5). Entre éstos se puede mencionar a todas las especies de Cantharidae y Carabidae las cuales solo estuvieron en las lluvias tanto a nivel del suelo, como en alguno de los estratos superiores; las especies de Coccinellidae se registraron solo a $1 \mathrm{~m}$, tanto en las lluvias como en sequía; las tres especies de Photinus (Lampyridae) se distribuyeron solo en los estratos superiores y únicamente se presentaron en las lluvias; Amecocerus sp. (Melyridae) durante las lluvias estuvo presente a $1 \mathrm{~m}$ y $5 \mathrm{~m}$, mientras que en la sequía solo se capturaron a nivel del suelo y a $1 \mathrm{~m}$; dentro de los Staphylinidae, las especies de Aleocharinae se concentraron en ambos estratos del dosel, contrario a lo ocurrido con la especie de Stilocharis sp. (Paederinae) y los Staphylininae: Belonuchus ephippiatus (Say, 1830), Heterotops sp. y Philonthus sp. que solo se recolectaron en el sotobosque (1 m) y exclusivamente en el periodo de lluvias.

Los fungívoros tuvieron su mayor riqueza de familias en el periodo húmedo y casi de manera exclusiva en los estratos superiores, con excepción de cuatro meses en los que Latridiidae y Mycetophagidae se recolectaron también a nivel del suelo. Por último, las familias xilófagas solamente se presentaron a finales de la sequía e inicio de las lluvias, exclusivamente en los estratos superiores y principalmente a $5 \mathrm{~m}$ (Figura 5).

\section{DISCUSIÓN}

El número de especies (126) y familias (31) registrado en los tres estratos está muy por debajo de la riqueza observada en áreas tropicales conservadas. Chung (2004) en una selva de tierras bajas en Malasia, registró 225 especies de 48 familias; Vega-badillo et al. (2018) en un bosque tropical caducifolio en México, obtuvieron 377 morfoespecies de 47 familias; Ulyshen y Hanula (2007) registraron 558 morfoespecies de 73 familias en un bosque templado caducifolio de Estados Unidos; Stork y Grimbacher (2006) registraron 1,473 especies de 77 familias en una selva tropical en Australia. La riqueza obtenida en el presente estudio es más parecida a la observada en áreas con climas templados, tal es el caso de Schroeder et al. (2009) quienes registraron 172 especies de 38 familias en un bosque templado de Canadá; Maguire et al. (2014) recolectaron 160 especies en un bosque caducifolio en Canadá; Kuprin y Drumont (2016) obtuvieron 135 especies y 32 familias en un bosque natural de olmo en Rusia.

El número total de familias registradas en los tres estratos en la Sierra de Guadalupe es inferior, si se compara con las 30 familias de coleópteros epigeos (estrato de 0 $\mathrm{m})$ registradas en las áreas de matorral xerófilo y pastizal ubicadas en la misma región donde se encuentra la Sierra de Guadalupe (García-Duran et al. 2013). Mientras que, en el presente estudio, en ese mismo estrato, únicamente se obtuvieron 12 familias; sin embargo, los valores de cobertura de la muestra indicaron que el muestreo es incompleto aún, por lo que un mayor esfuerzo de recolecta incrementará el número de especies. Esta suposición ha sido corroborada parcialmente con recolecciones no sistemáticas realizadas en el área de estudio, con red de golpeo y trampas de intercepción actuando durante más tiempo, con las cuales se obtuvieron cinco familias y 79 morfoespecies adicionales (datos no publicados). Por lo que el inventario total para el sitio de estudio se incrementaría a 36 familias y 205 morfoespecies. Estos hallazgos adicionales sugieren que esta porción de la Sierra, a pesar de la perturbación, puede albergar una riqueza mayor.

Más del 70\% de las especies en cada uno de los estratos fueron raras (singletons y doubletons), esto es algo común en la mayoría de las investigaciones; Stork y Grimbacher (2006) y Vega-badillo et al. (2018) encontraron que entre 30 y $40 \%$ de las especies por estrato estuvieron representadas por uno o dos individuos; Wagner (2000) al realizar fumigaciones del dosel registró 1,433 morfoespecies de escarabajos de las cuales $42 \%$ fueron singletons; Davis et al. (2011) encontraron un porcentaje de especies raras muy similar entre los estratos estudiados con un promedio entre ellos de $65 \%$. El caso más extremo fue el de Chung (2004) quien registró en el suelo $69.5 \%$ de singletons, $87 \%$ a 6 m y $96.6 \%$ a $12 \mathrm{~m}$. Estos dos últimos estudios tienen en común, un menor esfuerzo de recolección que las investigaciones de Vega-Badillo et al. (2018) y Stork y Grimbacher (2006) quienes muestrearon durante más tiempo y con mayor número de repeticiones; sin embargo, este artefacto del método de recolección es solo uno de los diferentes factores que causa el registro de especies raras, también podría tratarse de especies no residentes, fenología estacional, poblaciones cíclicas o verdadera rareza biológica (Hespenheide 2001). Por otra parte, las especies raras son importantes en el funcionamiento del ecosistema porque pueden reemplazar a especies comunes que se extinguen 
localmente al experimentar un crecimiento compensatorio (Jain et al. 2014). Es probable que esta parte perturbada de la Sierra, albergue muchas especies no residentes que provienen de la zona núcleo que se encuentra en la parte más alta, que por su difícil acceso aún conserva parte de la vegetación original (GCDMX 2016).

La diversidad beta fue debida principalmente al recambio, lo que se refleja también en un alto número de especies exclusivas de cada estrato. Este patrón ha sido observado en la mayoría de los estudios, con un elevado porcentaje de especies no compartidas entre los estratos, aunque en la mayoría de los casos éstas corresponden a especies raras (Grimbacher 2006; Ulyshen y Hanula 2007; Schroeder et al. 2009; Stork y Davis et al. 2011; Maguire et al. 2014; Kuprin y Drumont 2016; Vega-Badillo et al. 2018), lo cual podría ocultar el patrón exhibido por las especies comunes. Sin embargo, aun excluyendo a estas especies, el resultado no es una mezcla significativa de especies de los diferentes estratos, donde la distribución de especies raras puede ser consistente entre los diferentes niveles (Schroeder et al. 2009; Davis et al. 2011). Tal como ocurrió en el presente estudio donde alrededor del $70 \%$ de las especies fueron raras en cada uno de los estratos, aun al eliminarlas, se mantuvo el recambio espacial como el principal responsable de la diversidad beta. Esto sugiere una especialización por el estrato y, de acuerdo con Wardhaugh (2014), los patrones de agregación espacial y temporal de los insectos típicamente reflejan la distribución espacial y temporal de los recursos.

El sotobosque (estrato a $1 \mathrm{~m}$ ) aportó la mayor abundancia y la mayor cantidad de especies de coleópteros en la Sierra de Guadalupe, la mayoría de ellas exclusivas. Algunas familias con mayor riqueza de especies exclusivas de este estrato fueron: Cantharidae, Cerambicidae, Chrysomelidae, Coccinellidae, Curculionidae, Mordellidae, Scarabaeidae y Staphylinidae. Esto se acentúa con las diferencias significativas en los valores de diversidad obtenidos del sotobosque y el nivel del suelo con el dosel a $5 \mathrm{~m}$, que fue el más diverso debido a que tuvo una distribución más uniforme de la abundancia entre las especies. La preferencia de estos insectos por el sotobosque observada en la Sierra coincide con los resultados de otros estudios. Hill y Cermak (1997) encontraron que los artrópodos muestreados por medio de trampas de intercepción de vuelo fueron más abundantes (incluidos los coleópteros con más de 1,000 individuos en promedio capturados en el sotobosque, y solamente 73 y 49 individuos, a 5 y $10 \mathrm{~m}$ respectivamente) y posiblemente más diversos en las trampas más cerca del suelo que del dosel en una selva tropical. Por otra parte, Preisser et al. (1998) registraron mayor abundancia de insectos en el sotobosque que en el dosel de un bosque templado. Esa diferencia fue constante tanto para las capturas con trampas de luz como con las trampas malaise; aunque los autores señalan que no hubo una diferencia clara relacionada con la altura en el conteo total del orden Coleoptera. De igual manera, Kuprin y Drumont (2016) señalan que el estrato arbustivo (1 m de altura) contribuyó con la mayor riqueza de especies en un gradiente, el cual también incluyó el suelo a $0 \mathrm{~m}$ y el dosel a $11 \mathrm{~m}$; en el suelo dichos autores registraron mayor riqueza de especies (43) que en el presente estudio (19), aunque el número de familias fue inferior (7) con respecto a los registrados en la Sierra (12 familias), debido probablemente a que ellos emplean cinco veces más trampas pitfall que en el presente estudio, aunado a que el sitio de muestreo fue un bosque nativo sin impacto humano. En este sentido Su y Woods (2001) también observaron que hay una disminución de la riqueza de insectos con el incremento de la altura y que una gran proporción de la fauna de insectos es capturada más cerca del suelo incluso en los bosques talados sujetos a manejo. En la Sierra la presencia a nivel del sotobosque de una variada biomasa de plántulas de la vegetación secundaria con hojas nuevas, flores y semillas, la disponibilidad de troncos de árboles muertos y materia orgánica producida por la actividad humana son factores importantes para el incremento de la abundancia y riqueza en este estrato, debido a que la fisonomía del bosque y la arquitectura de la vegetación es determinante de la riqueza de insectos herbívoros asociados a los bosques tropicales (Basset et al. 2003).

Sin embargo, existe controversia sobre cuál es la contribución de cada estrato a la diversidad total, algunos autores encuentran que la contribución del dosel y el sotobosque son muy similares al no haber diferencias significativas entre éstos (Preisser et al. 1998; Stork y Grimbacher 2006; Ulyshen y Hanula 2007; Maguire et al. 2014; Vega-Badillo et al. 2018); mientras que otros han observado que el dosel aporta la mayor diversidad (Schroeder et al. 2009; Davis et al. 2011). En lo que coinciden todos los estudios, es en que sí existe una estratificación de la fauna de coleópteros. Estas inconsistencias reflejan la complejidad de las interacciones que pueden establecer los insectos con las plantas, con sus depredadores, su comportamiento, hasta artefactos del muestreo, lo que dificulta proponer una hipótesis que explique de la mejor manera los patrones de estratificación vertical (Wardhaugh 2014).

En la Sierra hubo una estacionalidad marcada con una mayor abundancia (81.8\%) y riqueza de especies $(62 \%)$ en el periodo de lluvias donde se presenta plantas jóvenes y brotes. Muchas de las especies fueron exclusivas de este periodo, en coincidencia con un valor alto del recambio de especies y un valor bajo de anidamiento. Este patrón ha sido observado en bosques templados donde los meses donde hay mayor abundancia de vegetación nueva disponible tienen los mayores registros de insectos en general (Preisser et al. 1998) y también de coleópteros de manera particular (Ulyshen y Hanula 2007; Hernández-Jáuregui et al. 2019). Ulyshen y Hanula (2007) lo atribuyen principalmente a un incremento en la calidad de los recursos como las hojas de las plantas jóvenes para los coleópteros herbívoros. En el presente estudio, los saprófagos, depredadores y fungívoros que fueron los gremios que agruparon más familias se presentaron todo el año, mientras que los herbívoros se 
concentraron principalmente en la época de lluvias.

En la Sierra, las familias se distribuyeron de manera diferente entre los estratos, Tenebrionidae fue la de mayor riqueza y de las más abundantes junto con Carabidae a nivel del suelo, aunque esta última tuvo mayor riqueza en el dosel; además, fue indicadora del suelo junto con Anthicidae, debido a que su frecuencia de captura fue mayor en este nivel. Esto concuerda con lo observado por Ulyshen y Hanula (2007), quienes registraron que los carábidos fueron más abundantes cerca del suelo $(0.5 \mathrm{~m})$ que en el dosel. En este último estrato también encontraron ejemplares de la tribu Lebiini, los cuales tuvieron una cantidad similar en ambos estratos, debido a que una de sus características es ser comunes en el dosel de bosques templados y tropicales. La familia Carabidae fue de las más diversas en los suelos de bosques templados (Kuprin y Drumont 2016) que es el estrato donde es más común su presencia. Cantharidae, Alleculinae (Tenebrionidae), Chrysomelidae, Latridiidae y Melolonthinae (Scarabaeidae) fueron más ricas y abundantes en el sotobosque; sin embargo, solo estas últimas tres, así como, Coccinellidae, Mordellidae y Ptinidae resultaron como indicadoras del estrato de $1 \mathrm{~m}$. En el dosel se encontró a Scolytinae (Curculionidae), Aleocharinae (Staphyinidae) y Bruchinae (Chrysomelidae), de las cuales las últimas dos fueron indicadoras de este estrato por su mayor frecuencia de captura. Lo anterior concuerda con la distribución observada en otros estudios para cada una de estas familias (Chung 2004; Stork y Grimbacher 2006; Ulyshen y Hanula 2007; Maguire et al. 2014; Kuprin y Drumont 2016; Vega-Badillo et al. 2018). En este estudio también destacó Cerambycidae por su preferencia por el dosel a $1 \mathrm{~m}$ y $5 \mathrm{~m}$; las especies de esta familia se destacan por ser trozadoras y es frecuente encontrarlas en el dosel sobre ramas de diámetro variable (V. H. Toledo com. pers). Maguire et al. (2014) también observaron el mismo patrón para Cerambycidae y Bruchinae. Por otra parte, Scolytinae (Curculionidae) fue capturada en todas las repeticiones de $1 \mathrm{~m}$ y $5 \mathrm{~m}$ en este estudio (Cuadro 5), a diferencia de Ulyshen y Hanula (2007) quienes encontraron que los escarabajos descortezadores (Scolytinae) fueron más abundantes y ricos en especies cerca del suelo que en el dosel. Sin embargo, en este mismo estudio miembros del género Pityophthorus (Scolitynae) que atacan las ramas de los árboles fueron significativamente más abundantes en el dosel. Lo anterior se debe a que las especies de Scolytinae realizan vuelos a poca altura, pero infestan ramas en todos los estratos; lo cual depende de la atracción ejercida por las ramas de acuerdo con su condición (V. H. Toledo com. pers.). De manera general la preferencia de estas familias en este y otros estudios apoyan la hipótesis de la disponibilidad del recurso propuesta por Wardhaugh (2014), que establece que muchos insectos especialistas del estrato están probablemente restringidos a un estrato particular simplemente porque su fuente de alimento está también restringida o concentrada en el mismo estrato.

A diferencia de los casos anteriores, dentro de cada familia hay especies que son más abundantes en el sotobosque y otras que son más comunes en el dosel, como ha sido observado en las especies de Cerambycidae (Vance et al. 2003; Ulyshen y Hanula 2007), Scarabaeidae (Vulinec et al. 2007) o Coccinellidae, que de manera particular tienen variaciones de la actividad del vuelo dentro de cada especie y entre los años, debido probablemente a la abundancia relativa de las presas y de una combinación particular de cultivos o plantas (Boiteau et al. 1999). Por lo que, es claro que la distribución diferencial vertical está determinada por varios factores tanto bióticos como abióticos (Ulyshen 2011), como son la disponibilidad de recursos o el régimen pluviométrico. Durante la sequía se acumula materia orgánica en descomposición que proviene principalmente de las plantas herbáceas anuales o de animales muertos, lo cual puede explicar que las especies saprófagas de Anthicidae sp. 1 y Tenebrionidae spp. durante esta época se concentraron en el suelo, y en las lluvias las especies de Hymenorus spp. (Tenebrionidae: Alleculinae) y Anthicidae sp. 1 prefirieron los estratos superiores de la vegetación. Aunque, las especies saprófagas de Anotylus sp. y Platystethus sp. (Staphylinidae: Oxytelinae) estuvieron en todos los estratos, prefirieron el dosel durante la sequía.

Por el contrario, la disponibilidad de follaje nuevo y flores, influyó en que los herbívoros estuvieran exclusivamente en el estrato arbustivo y el dosel, con una mayor riqueza de familias en el periodo de lluvias. Por ejemplo, los adultos de Melolonthinae y Cetoniinae (Scarabaeidae) que fueron abundantes en el sotobosque $(1 \mathrm{~m})$. Para estos escarabajos herbívoros la estratificación puede ser compleja, los adultos se alimentan y buscan pareja en diferentes niveles del dosel, mientras que las larvas viven en el suelo alimentándose de raíces (Basset et al. 2003). Una distribución similar se observó para estos grupos de lamelicornios en un bosque templado, aunque con la especie Euphoria fulgida (Fabricius, 1775) que fue exclusiva del dosel a más de $15 \mathrm{~m}$, esto debido a que los cetoninos se alimentan principalmente de líquidos como néctar o savia que encuentran en la parte superior de los bosques (Ulyshen y Hanula 2007).

Por otra parte, los depredadores estuvieron en todos los estratos durante todo el año, con una abundancia ligeramente mayor en la parte superior del bosque. Por ejemplo, los estafilínidos de las subfamilias Paederinae (Stilocharis sp.) y Staphylininae (Belonuchus ephippiatus (Say, 1830), Heterotops sp. y Philonthus sp.) solo se recolectaron en el sotobosque y exclusivamente en el periodo de lluvias, estos últimos acuden principalmente a la materia orgánica en descomposición para buscar a sus presas (Navarrete-Heredia et al. 2002), por lo que no es raro que solo vuelen a nivel de sotobosque buscando excremento, carroña o plantas en descomposición acumuladas en el suelo. De manera general la presencia de los depredadores en todos los estratos se debe a que éstos son muy activos en la búsqueda de sus presas, esto coincide con lo observado en un bosque nativo de olmo conservado, excepto que la abundancia de dicho 
gremio fue mayor en el suelo (0 m) (Kuprin y Drumont 2016) debido a que la mitad de las especies en este estrato correspondieron a la familia Carabidae los cuales son muy activos en el suelo de los bosques templados, por lo que los hábitos de las especies predominantes en cada sitio influyen en la determinación general de los patrones observados.

Los fungívoros tuvieron su mayor riqueza en el periodo húmedo y prefirieron los estratos superiores, tal es el caso de las familias Latridiidae y Mycetophagidae, que fueron más abundantes en el sotobosque y el dosel, la primera de estas familias también puede incluir algunas especies herbívoras, en la Sierra estuvo presente tanto en lluvias como en sequía y su distribución vertical coincidió con lo observado en otros estudios donde capturan a Latridiidae y otros grupos de micófagos principalmente en el dosel de bosques templados (Ulyshen y Hanula 2007; Kuprin y Drumont 2016) y tropicales (Wagner 2000); por otra parte Melandrydae sp. 1 y Ptilidae sp. se capturaron exclusivamente en los estratos superiores en ambos periodos del año. Lo anterior sugiere que el moho y otros hongos pueden ser un importante recurso en el dosel de muchos bosques.

De acuerdo con la información que se tiene acerca de los hábitos alimentarios de las familias capturadas y su periodo de captura, la distribución vertical se puede explicar como el resultado de la interacción entre la disponibilidad de los recursos alimentarios y la estacionalidad climática. Wardhaugh (2014) menciona a la disponibilidad del recurso como el factor más importante responsable en la determinación de los patrones de estratificación vertical, distribución horizontal y en algunos casos estacional y de actividad diaria de los ensambles de insectos arbóreos, los cuales en gran medida reflejan dónde y cuándo están disponibles los recursos apropiados.

La fauna de coleópteros del bosque artificial de la Sierra de Guadalupe muestra los patrones de distribución vertical y estacional característicos de otros bosques templados y tropicales, así como de la composición de los gremios tróficos. La riqueza fue más parecida a la observada en algunos estudios con bosques templados, aunque los estimadores y los muestreos realizados con otros métodos de recolecta en la Sierra, sugieren que hace falta un mayor esfuerzo de recolecta.

La diferencia en la distribución vertical de la fauna de coleópteros observada en la Sierra de Guadalupe puede ser explicada por la distribución espacial de los recursos alimentarios, mientras que su variación temporal estaría relacionada con las condiciones de estacionalidad climática; sin embargo, no es la única fuerza responsable de la estratificación de la diversidad. Otras causas pueden ser las interacciones de competencia y depredación entre las especies, comportamiento o su historia evolutiva, las cuales tendrían que ser evaluadas en estudios más específicos (Wardhaugh 2014).

La alteración de la estructura original de la vegetación generó nuevos microhábitats que favorecieron una composición distinta a la que se encontraba en la vegetación original del área, la cual se ha intentado recuperar con reforestaciones de áreas pequeñas pero múltiples, utilizando especies de árboles no nativas desde hace más de 50 años (GCDMX 2016). Este estudio muestra que, a pesar de la perturbación, el área alberga una riqueza importante de diferentes gremios tróficos que a lo largo del tiempo han establecido redes que mantienen el funcionamiento del ecosistema.

Los resultados obtenidos servirán como punto de comparación con estudios posteriores que pueden ser llevados a cabo en la zona núcleo de la Sierra, que aún conserva la vegetación nativa. Se esperaría que en esa área hubiera diferencias importantes en la estructura y composición de los ensambles de coleópteros con respecto a lo encontrado en el presente estudio, para de esta manera tratar de conocer el estado de salud y recuperación que ha tenido el área. Es importante que el estrato del dosel sea considerado en los programas de manejo y conservación, para tratar de recuperar el dosel del hábitat original mediante la sustitución paulatina de las especies de árboles exóticas.

Finalmente, como en la mayoría de los estudios, resalta el papel tan importante que tienen los diferentes estratos de vegetación en la determinación de la riqueza de coleópteros y en general de la fauna de artrópodos del área, los cuales contribuye a la estructura, fertilidad, y dinámica espacial, y son un elemento crucial para mantener la biodiversidad y las redes tróficas (Cardoso et al. 2020).

\section{AGRADECIMIENTOS}

Este estudio fue parcialmente apoyado por el Proyecto Zoología de la División de Investigación y Posgrado de la FES Iztacala, UNAM. A Mónica Chico Avelino por la elaboración del mapa. A Itzel Rodríguez Castillo, Andrés Oropeza Petrich, Mónica Copihue Vega Ávila, Raquel Castillo Flores y Daniel G. Torres Millán, así como, a los guardaparques del Parque Estatal Sierra de Guadalupe por su apoyo durante los muestreos. A Álvaro Fuentealba Morales por la revisión del abstract. A Cisteil Xinum Pérez Hernández y a un revisor anónimo por sus valiosos comentarios que permitieron mejorar el escrito.

\section{LITERATURA CITADA}

Arnett, Jr., R.H, and M.C. Thomas. 2001. Volume 1. American Beetles. Archostemata, Myxophaga, Adephaga, Polyphaga: Staphylinifromia. CRC Press, EU.

Arnett, Jr., R.H., M.C. Thomas, P.E. Skelley and J.H. Frank. 2002. Volume 2. American Beetles. Polyphaga: Scarabaeoidea through Curculionoidea. CRC Press, EU.

Baselga, A. 2010. Partitioning the turnover and nestedness components of beta diversity. Global Ecology and Biogeography 19(1): 134-143.

Basset, Y. 1988. A composite interception trap for sampling arthropods in tree canopies. Australian Journal of Entomology 27(3): 213-219. https://doi. 
org/10.1111/j.1440-6055.1988.tb01527.x.

Basset, Y., H.P. Aberlenc and G. Delvare. 1992. Abundance and stratification of foliage arthropods in a lowland rain foresto of Cameroon. Ecological Entomology 17(4): 310-318.

Basset, Y., H.P. Aberlenc, H. Barrios, G. Curletti, J.M. Bérenger, J. P. Vesco, P. Causse. R. Haug, A.S. Hennion, L. Lesobre, F. Marqueás and R. Omeara. 2001. Stratification and diel activity of arthropods in a lowland rainforest in Gabon. Biological Journal of the Linnean Society 72(4): 585-607. https://doi. org/10.1006/bij1.2001.0518.

Basset, Y., P.M. Hammond, H. Barrios, J.D. Holloway and S. E. Miller. 2003. Vertical stratification of arthropod assemblages. (pp. 17-27). In: Basset, Y., V. Novotny, S.E., Miller and R.L., Kitching (Eds.) Arthropods of tropical forest; spatio- temporal dynamics and resource use in the canopy. Cambridge, Cambridge University Press.

Boiteau, G., Y. Bousquet and W.P.L. Osborn. 1999. Vertical and temporal distributios of Coccinellidae (Coleoptera) in flight over an agricultural landscape. The Canadian Entomologist 131(2): 269-277.

Bouchard, P., Y. Bousquet, A.E. Davies, M.A. AlonsoZarazaga, J.F. Lawrence, C.H.C. Lyal, A.F. Newton, C.A.M. Reid, M. Schmitt, S.A. Slipiński and A.B.T.Smith. 2011. Family-group names in Coleoptera (Insecta). ZooKeys 972(88): 1-972. https://doi. org/10.3897/zookeys.88.807.

Cedillo, O.L., M.Á. Rivas y F.N. Rodríguez. 2007. El Área Natural Protegida sujeta a conservación ecológica "Sierra de Guadalupe". Revista Sistemas Ambientales 1(1): 1-14.

Chao, A., N.J. Gotelli, T.C. Hsieh, E.L. Sander, K.H. Ma, R.K. Colwell and A.M. Ellison. 2014. Rarefaction and extrapolation with hill numbers : a framework for sampling and estimation in species diversity studies. Ecological Monographs 84(1): 45-67. https://doi. org/10.1890/13-0133.1.

Chung, A. 2004. vertical stratification of beetles (Coleoptera) using flight intercept traps in a lowland rainforest of Sabah, Malaysia. Sepilok Bulletin (1): 27-39.

Davis, A. J, S. L. Sutton and M.J.D. Brendell. 2011. Vertical distribution of beetles in a tropical rainforest in Sulawesi: The Role of the Canopy in Contributing to Biodiversity. Sepilok Bulletin 13 and 14: 59-83.

De Caceres M. and P. Legendre. 2009. Association between species and groups of sites indices and statistical inference. Ecology 90(12): 3566-3574.

Dufrêne, M. and P. Legendre. 1997. Species assemblages and indicator species: the need for a flexible asymmetrical approach. Ecological Monographs 67(3): 345-366.

García-Durán, A., E. Jiménez-Sánchez y J. PadillaRamírez. 2013. Coleoptera (Insecta) del suelo de una región semiárida en el nororiente del Estado de México, México. (pp. 1619-1624). En: Equihua, A., E.G.
Estrada, J.A. Acuña y M.P. Chaires (Eds.). Entomología Mexicana Vol. 12. Tomo 2. Sociedad Mexicana de Entomología A.C., México.

Grimbacher, P.S. and N.E. Stork. 2007. Vertical stratification of feeding guilds and body size in beetle assemblages from an australian tropical rainforest. Austral Ecology 32(1): 77-85. https://doi.org/10.1111/ j.1442-9993.2007.01735.x.

Hernández-Jáuregui, M., M.R. Gómez-Pastrana, R. SernaLagunes, C. Llarena-Hernández y N. Mora-Collado. 2019. Diversidad estacional y vesticalde familias del orden coleoptera en un fragmento de bosque de pinooyamel, Tlaxcala, México. Tropical and Subtropical Agroecosystems 22(2): 401-408.

Hespenheide, H.A. 2001. Beetles. (pp. 351-358). In: Levin, S.A. (Ed.). Encyclopedia of biodiversity. Academic Press, California, USA. https://doi.org/10.1016/B0-12226865-2/00025-0.

Hill, C.J. and M. Cermak. 1997. A new design and some preliminary results for a flight intercept trap to sample forest canopy arthropods. Australian Journal of Entomology 36(1): 51-55. https://doi. org/10.1111/j.1440-6055.1997.tb01431.x.

Hsieh, T.C., K.H. Ma and A. Chao. 2016. iNEXT: An R package for rarefaction and extrapolation of species diversity (Hill numbers). Methods in Ecology and Evolution 7(12): 1451-1456. DOI: 10.1111/2041210X.12613

Jain, M., D.F.B. Flynn, C.M. Prager, G.M. Hart, C.M. DeVan, F.S. Ahrestani, M.I. Palmer, D.E. Bunker, J.M.H. Knops, C.F. Jouseau and S. Naeem. The importance of rare species: a trait-based assessment of rare species contributions to functional diversity and possible ecosystem function in tall-grass prairies. Ecology and Evolution, 4(1): 104-112. doi: 10.1002/ece3.915

Jiménez-Sánchez, E., R. Quezada-García, J. PadillaRamírez, M.L. Moreno y M.A. Angel. 2019. Variación de la diversidad de Staphylinidae, Silphidae y Trogidae (Insecta: Coleoptera) en un gradiente urbano-agrícola en una región semiárida del Estado de México, México. Acta Zoológica Mexicana (N.S.), 35: 1-16. https://doi. org/10.21829/azm.2019.3502152.

Kato, M., T. Inoue, A.A. Hamid, T. Nagamitsu, M.B. Merdek, A.R. Nona, T. Itino, S. Yamame, and T. Yumoto. 1995. Seasonality and vertical structure of light-attracted insect communities in a dipterocarp forest in Sarawak. Research on Population Ecology 37(1): 59-79.

Kuprin, A.V. and A. Drumont. 2016. Stratification and diversity of beetles (insecta, coleoptera) in native elm forests of the Ussuri Nature Reserve, Russia. Entomology and Applied Science Letters 3(6): 1-8.

Lugo-Hubp, J. y A. Salinas-Montes. 1996. Geomorfología de la Sierra de Guadalupe (al norte de la Ciudad de México) y su relación con peligros naturales. Revista Mexicana de Ciencias Geológicas 13(2): 240-251. 
Maguire, D.Y., K. Robert, K. Brochu, M. Larrivée, C.M. Buddle and T.A. Wheeler. 2014. Vertical stratification of beetles (Coleoptera) and flies (Diptera) in temperate forest canopies. Environmental Entomology 43(1): 9-17. https://doi.org/10.1603/EN13056.

Moreno, C.E., J.M. Calderón-Patrón, N. Martín-Regalado, A.P. Martínez-Falcón, I.J. Ortega-Martínez, C.L. RiosDíaz, and F. Rosas. 2018. Measuring species diversity in the tropics : a review of methodological approaches and framework for future studies. Biotropica, 50(6): 929-941. https://doi.org/10.1111/btp.12607.

GCDMX (Gobierno de la Ciudad de México). 2016. Programa de manejo del área natural protegida, con categoría de zona sujeta a conservación ecológica "Sierra de Guadalupe". Gaceta Oficial de La Ciudad de México, 196: 1-136.

Navarrete-Heredia, J. L., A.F. Newton, M.K. Thayer, J.S. Ashe and D.S. Chandler. 2002. Guía Ilustrada Para Los Géneros de Staphylinidae (Coleoptera) de México. Universidad de Guadalajara y CONABIO, México.

Navarrete-Heredia, J.L., C.I. Sainz, A.L. GonzálezHernández, G.A. Quiroz-Rocha, A. Hernández, M. Vásquez-Bolaños, D. Vega-Romero y B. Hernández. 2012. Coleópteros necrócolos del bosque Los Colomos, Guadalajara, Jalisco, México. Dugesiana 19(2): 157162.

Peña, A.K. 2018. Análisis del cambio espacio-temporal de la cobertura vegetal dentro de la Sierra de Guadalupe, México, mediante teldetección. Tesis de maestría, Instituto Politécnico Nacional, México.

Preisser, E., D.C.Smith and M. Lowman. 1998. Canopy and ground level insect distribution in a temperate forest. Selbyana 19(2): 141-146.

R Development Core Team. 2019. R: a language and environment for statistical computing. Version 3.5.2. Viena, Austria: R Foundation for Statistical Computing. http://www.R-project.org. Fecha de consulta: noviembre 2019.

Schroeder, B., C.M. Buddle and M. Saint-Germain. 2009. Activity of flying beetles (Coleoptera) at two heights in canopy gaps and intact forests in a hardwood forest in Quebec. Canadian Entomologist 141(5): 515-520. https://doi.org/10.4039/n09-022.

Stork, N.E. and P.S. Grimbacher. 2006. Beetle assemblages from an australian tropical rainforest show that the canopy and the ground strata contribute equally to biodiversity. Proceedings of the Royal Society B: Biological Sciences 273(1596): 1969-1975. https://doi. org/10.1098/rspb.2006.3521.

$\mathrm{Su}$, J.C. and S.A. Woods. 2001. Importance of sampling along a vertical gradient to compare the insect fauna in managed forests. Environmental Entomology 30(2): 400-408. https://doi.org/10.1603/0046-225X-30.2.400.
Schwartz, M.W., C.A. Brigham, J.D. Hoeksema, K.G. Lyons, M.H. Mills, and P.J. Van Mantgem. 2000. Linking biodiversity to ecosystem function: implications for conservation ecology. Oecologia, 122(3): 297-305. https://doi.org/10.1007/s004420050035.

Triplehorn, C.A. and N.F. Johnson. 2005. Borror and Delong's introduction to the study of insects. Thomson Brooks/Cole, EU.

Ulyshen, M.D. 2011. Arthropod vertical stratification in temperate deciduous forests: implications for conservation-oriented management. Forest Ecology and Management 261(9): 1479-1489. https://doi. org/10.1016/j.foreco.2011.01.033.

Ulyshen, M.D., S. Horn and J.L. Hanula. 2010. Response of beetles (Coleoptera) at three heights to the experimental removal of an invasive shrub, chinese privet (Ligustrum sinense), from floodplain forests. Biological Invasions 12(6): 1573-1579. https://doi.org/10.1007/s10530-0099569-2.

Ulyshen, M.D. and J.L. Hanula. 2007. A comparison of the beetle (Coleoptera) fauna captured at two heights above the ground in a North American temperate deciduous forest. The American Midland Naturalist 158(2): 260278.

Vance, C.C., K.R. Kirby, J.R. Malcolm and S.M. Smith. 2003. Community composition of longhorned beetles (Coleoptera: Cerambycidae) in the canopy and understorey of sugar maple and white pine stands in south-central Ontario. Environmental Entomology 32(5): 1066-1074. https://doi.org/10.1603/0046225X-32.5.1066.

Vega-Badillo, V., S. Zaragoza-Caballero, C. Moreno and I. Trejo. 2018. Distribución vertical de Coleoptera (Insecta) en un bosque tropical subcaducifolio en Jalisco, México. Revista Mexicana de Biodiversidad 89(3): 836-846.

Vulinec, K., D.J. Mellow and C.R. Vasconcellos da Fonseca. 2007. Arboreal foraging height in a common neotropical dung beetle, canthon subhyalinus harold (Coleoptera: Scarabaeidae). Coleopterists Bulletin 61(1): 75-81. https://doi.org/10.1649/915.1.

Wagner, T. 2000. Influence of forest type and tree species on canopy-dwelling beetles in budongo forest, Uganda. Biotropica 32(3): 502-514. https://doi. org/10.1111/j.1744-7429.2000.tb00496.x.

Wardhaugh, C. W. 2014. The spatial and temporal distributions of arthropods in forest canopies : uniting disparate patterns with hypotheses for specialisation. Biological Reviews, 89(4): 1021-1041. https://doi. org/10.1111/brv.12094.

Zhang, Z.-Q. 2013. Phylum Arthropoda. Zootaxa 3703(1): 17-26. 

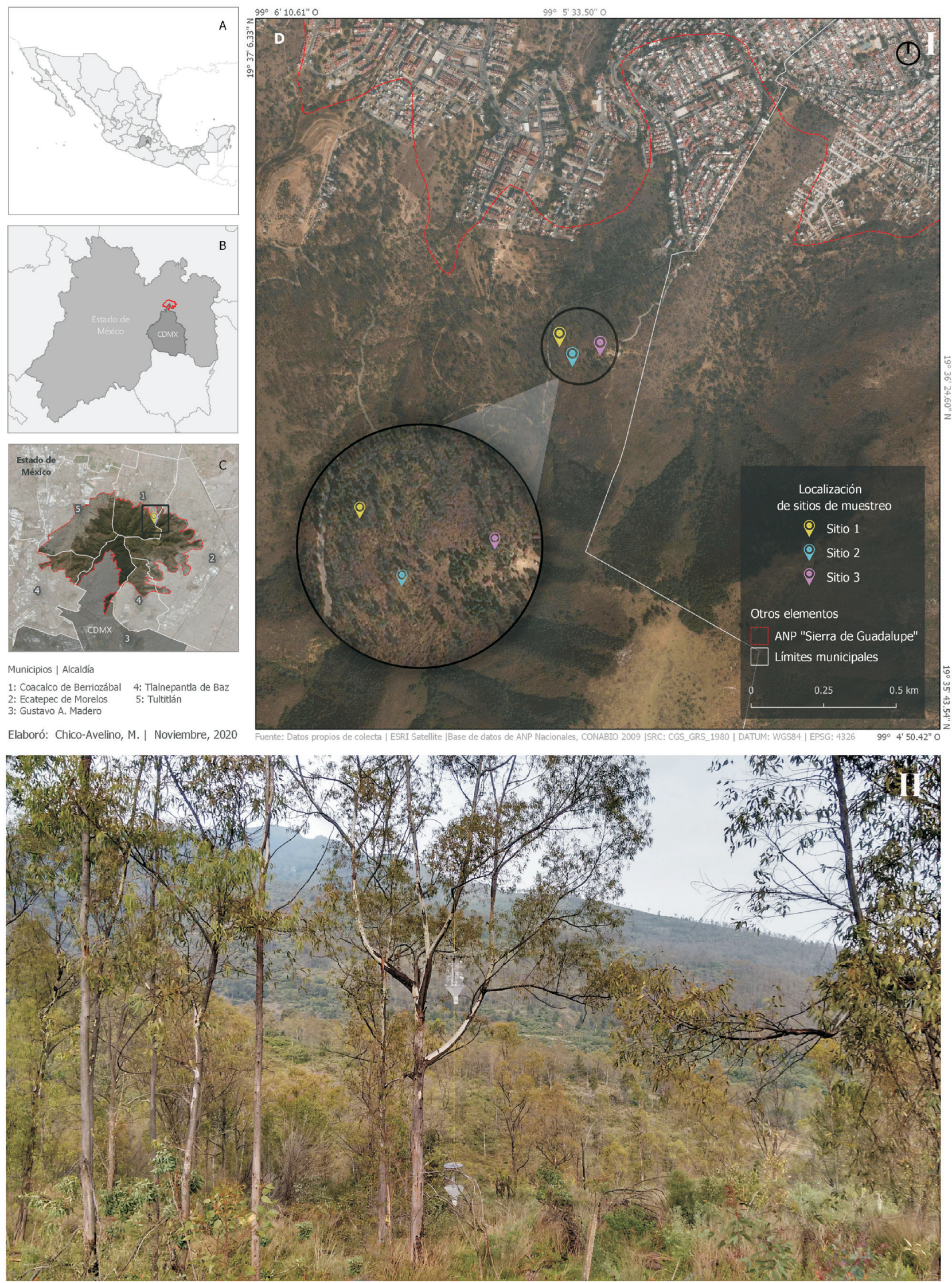

Figura 1. I. Ubicación del Área de estudio. Nivel nacional (A), nivel estatal (B), perspectiva de la Sierra de Guadalupe (C) y sitios de recolecta (D). II. Panorámica del hábitat donde se realizaron los muestreos. Foto por E. Jiménez-Sánchez. 


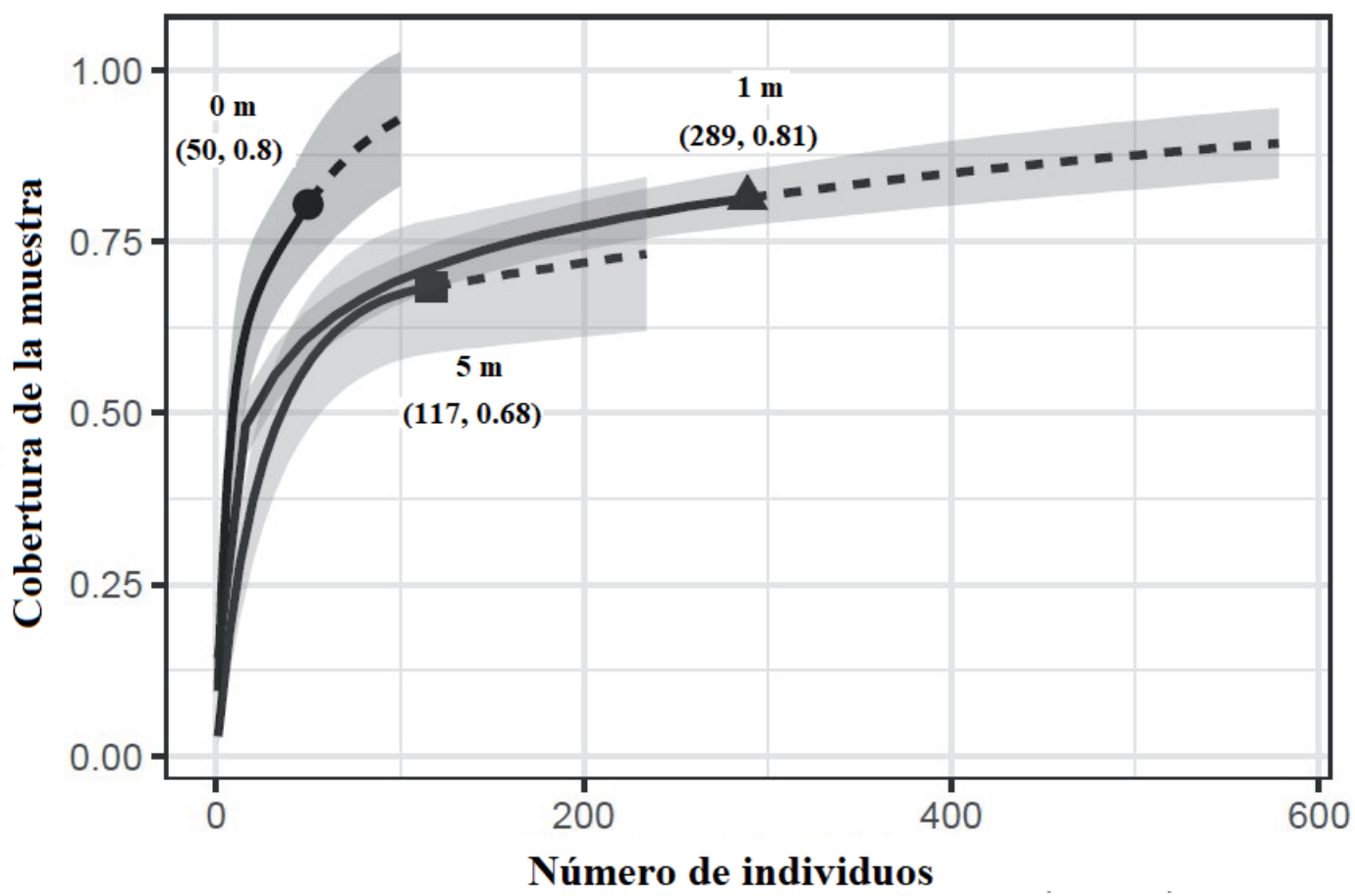

Figura 2. Curva de cobertura de la muestra, para la muestra de referencia (línea continua) y la muestra extrapolada (línea discontinua) de los coleópteros capturados en tres diferentes estratos de vegetación en un bosque artificial de la Sierra de Guadalupe.
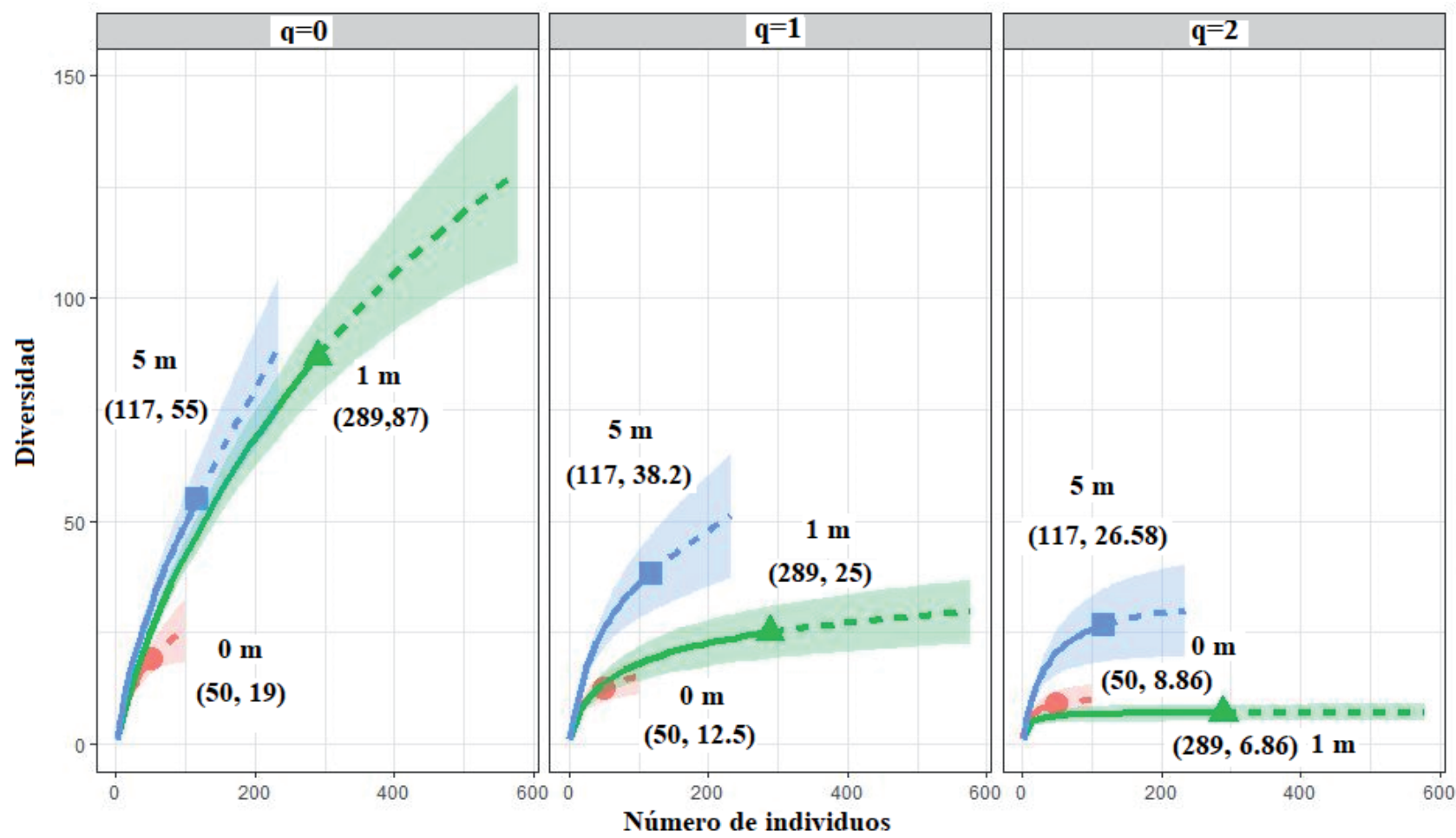

Figura 3. Comparación de las curvas de rarefacción (línea continua) - extrapolación (línea discontinua) basadas en el tamaño de la muestra de la diversidad de especies de coleópteros para los números de Hill del orden q=0 (izquierda), q=1 (centro) y q=2 (derecha). Las muestras de referencia para cada altura se indican con figuras: $0 \mathrm{~m}$ (círculo), $1 \mathrm{~m}$ (triángulo) y $5 \mathrm{~m}$ (cuadro). Los números en paréntesis son el tamaño de la muestra y los números de Hill observados respectivamente para cada muestra de referencia. 

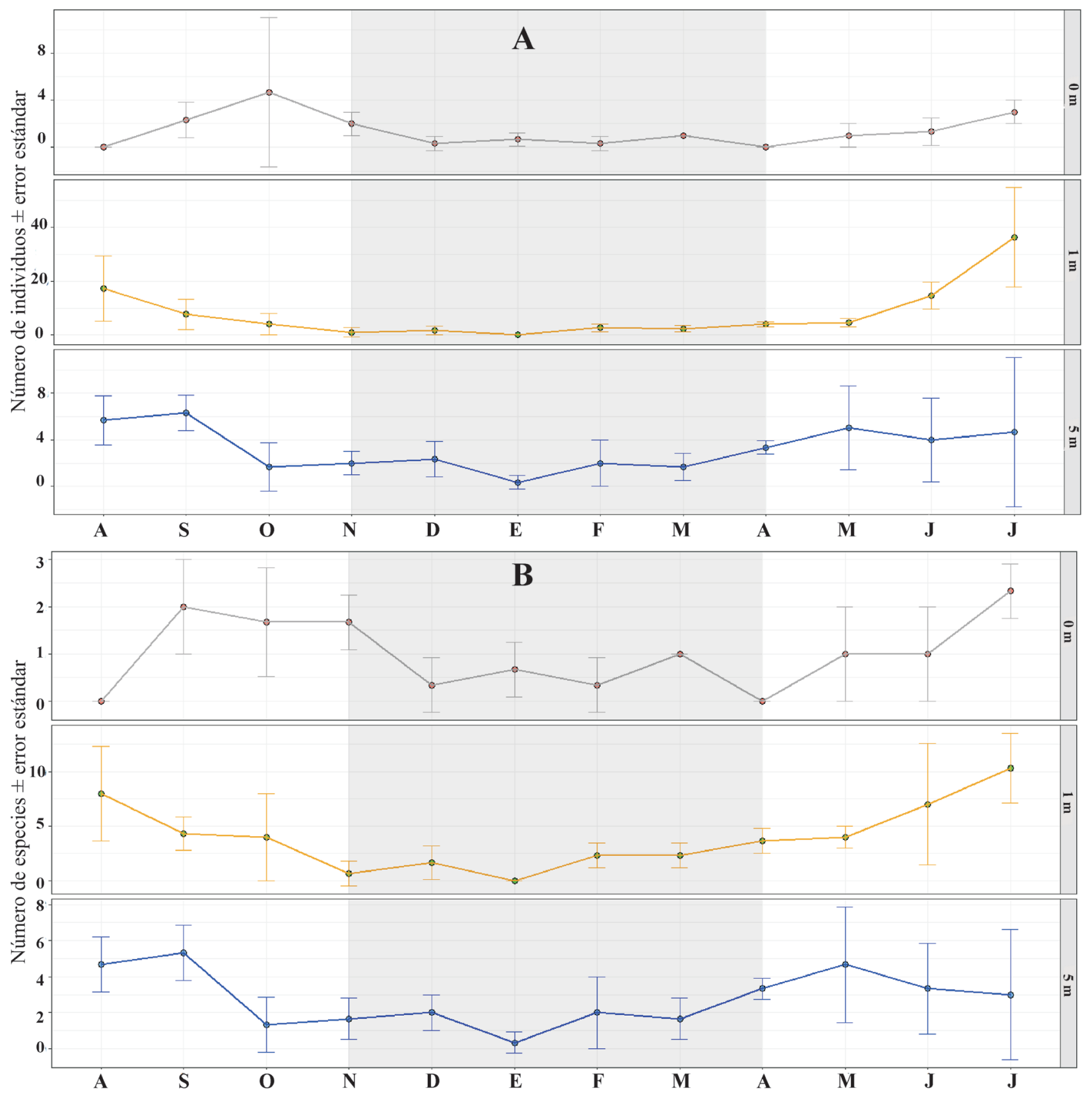

Figura 4. Promedio mensual \pm error estándar del número de individuos (A) y especies (B) de coleópteros capturados en tres diferentes alturas en un bosque artificial $(n=3)$ de la Sierra de Guadalupe. Los meses sombreados corresponden a la temporada de sequía. 

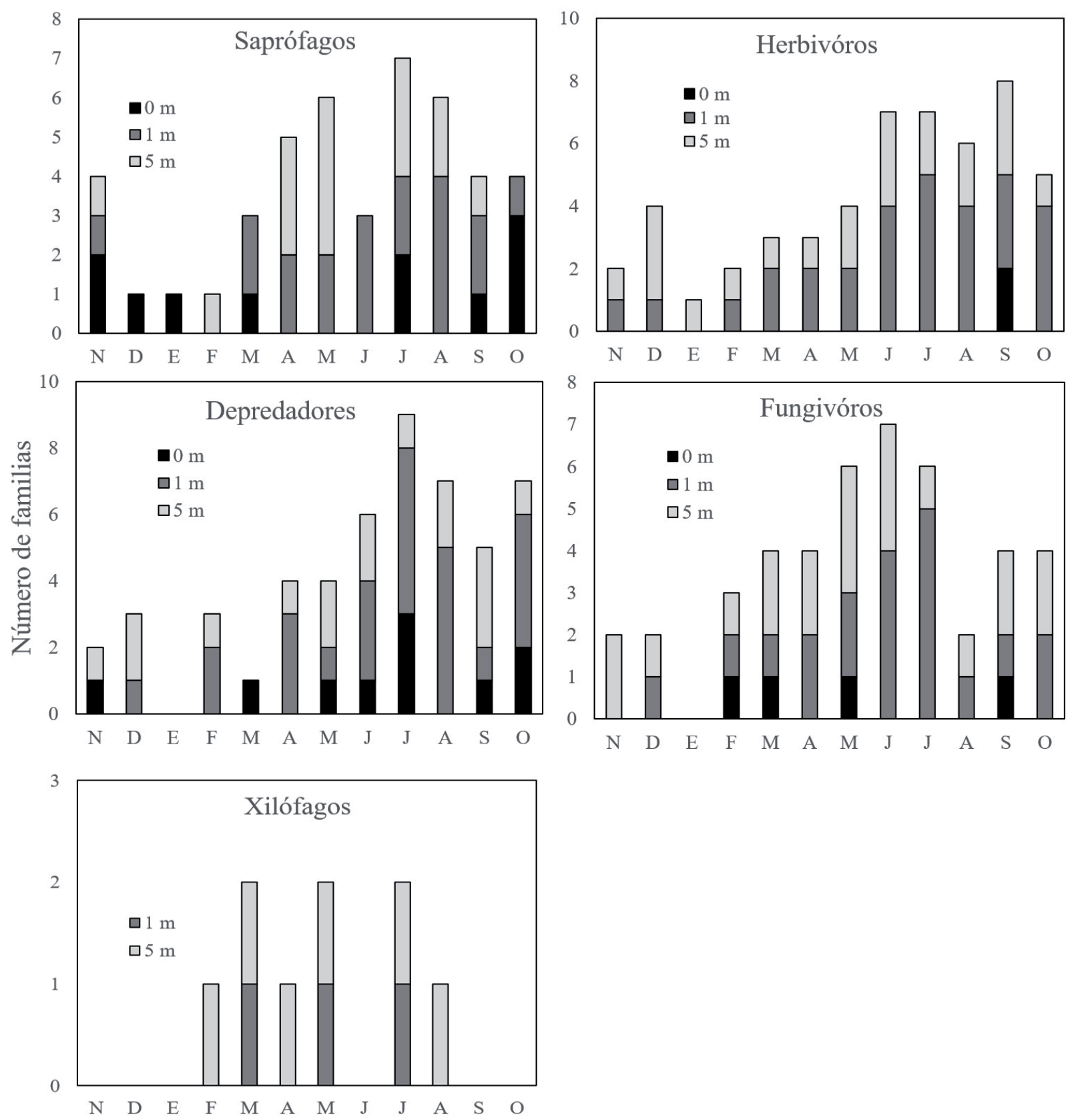

Figura 5. Número de familias del orden Coleoptera capturadas por mes, para cada gremio trófico en tres diferentes estratos en un bosque artificial de la Sierra de Guadalupe. 
Cuadro 1. Lista de familias y número de especies e individuos capturados en tres diferentes estratos en un bosque artificial de México. $\mathrm{H}=$ herbívoro; $\mathrm{P}=$ depredador; $\mathrm{F}=$ fungívoro; $\mathrm{X}=$ xilófago; $\mathrm{S}=$ saprófago; ? $\mathrm{P}=$ posible depredador; $\mathrm{P},(\mathrm{H})=$ la mayoría de las especies de la familia son depredadoras, pero algunas especies son herbívoras; ? = gremio trófico desconocido. Las familias y los números en negritas indican que el taxón está en todos los estratos o que es exclusivo del estrato respetivamente. *Chrysomelidae $\sin$ Bruchinae. ${ }^{* *}$ Curculionidae sin Scolytinae. $* * *$ Tenebrionidae sin Alleculinae.

\begin{tabular}{|c|c|c|c|c|c|c|c|c|c|}
\hline \multirow[b]{2}{*}{ Familia } & \multicolumn{3}{|c|}{ No. de especies } & \multicolumn{5}{|c|}{ No. de individuos } & \multirow{2}{*}{$\begin{array}{c}\text { Gremio } \\
\text { trófico }\end{array}$} \\
\hline & $\mathbf{0 m}$ & $1 \mathrm{~m}$ & $5 \mathrm{~m}$ & Total & $\mathbf{0 m}$ & $1 \mathrm{~m}$ & $5 \mathrm{~m}$ & Total & \\
\hline Anthicidae & 1 & 1 & 0 & 1 & 6 & 1 & 0 & 7 & $\mathrm{~S}, ? \mathrm{P}$ \\
\hline Anthribidae & 0 & 0 & 1 & 1 & 0 & 0 & 1 & 1 & $\mathrm{~F},(\mathrm{H})$ \\
\hline Cantharidae & 1 & 3 & 0 & 4 & 1 & 10 & 0 & 11 & $\mathrm{P},(\mathrm{H})$ \\
\hline Carabidae & 2 & 0 & 4 & 5 & 12 & 0 & 6 & 18 & $\mathrm{P}$ \\
\hline Cerambycidae & 0 & 2 & 1 & 3 & 0 & 2 & 1 & 3 & $\mathrm{X},(\mathrm{H})$ \\
\hline Chrysomelidae* & 1 & 8 & 5 & 12 & 2 & 14 & 5 & 21 & $\mathrm{H}$ \\
\hline Bruchinae & 0 & 6 & 5 & 7 & 0 & 9 & 12 & 21 & $\mathrm{H}$ \\
\hline Cleridae & 1 & 2 & 0 & 2 & 2 & 2 & 0 & 4 & $\mathrm{P},(\mathrm{H})$ \\
\hline Coccinellidae & 0 & 5 & 0 & 5 & 0 & 7 & 0 & 7 & $\mathrm{P},(\mathrm{H})$ \\
\hline Cucujidae & 0 & 1 & 0 & 1 & 0 & 1 & 0 & 1 & $\mathrm{~F},(\mathrm{P})$ \\
\hline Curculionidae ${ }^{* *}$ & 2 & 4 & 4 & 8 & 2 & 6 & 6 & 14 & $\mathrm{H},(\mathrm{X})$ \\
\hline Scolytinae & 0 & 3 & 5 & 7 & 0 & 3 & 7 & 10 & $\mathrm{X}, \mathrm{X} / \mathrm{F}$ \\
\hline Dascillidae & 0 & 1 & 0 & 1 & 0 & 3 & 0 & 3 & $?$ \\
\hline Elateridae & 0 & 2 & 0 & 2 & 0 & 2 & 0 & 2 & $\mathrm{H}, \mathrm{X}, \mathrm{P}, \mathrm{S}$ \\
\hline Histeridae & 0 & 1 & 0 & 1 & 0 & 2 & 0 & 2 & $\mathrm{P}$ \\
\hline Hydrophilidae & 0 & 0 & 1 & 1 & 0 & 0 & 1 & 1 & $\mathrm{~S},(\mathrm{P})$ \\
\hline Lampyridae & 0 & 2 & 2 & 3 & 0 & 4 & 2 & 6 & $\mathrm{P}$ \\
\hline Latridiidae & 1 & 2 & 1 & 2 & 4 & 18 & 10 & 32 & $\mathrm{~F},(\mathrm{H})$ \\
\hline Leiodidae & 0 & 0 & 1 & 1 & 0 & 0 & 1 & 1 & $\mathrm{~F}$ \\
\hline Melandrydae & 0 & 2 & 1 & 2 & 0 & 5 & 5 & 10 & $\mathrm{~F},(\mathrm{X})$ \\
\hline Meloidae & 0 & 1 & 0 & 1 & 0 & 2 & 0 & 2 & $\mathrm{H}$ \\
\hline Melyridae & 1 & 2 & 1 & 2 & 1 & 8 & 3 & 12 & $\mathrm{P}$ \\
\hline Monotomidae & 2 & 2 & 1 & 4 & 3 & 2 & 1 & 6 & $\mathrm{~S}, \mathrm{~F}$ \\
\hline Mordellidae & 0 & 4 & 1 & 4 & 0 & 6 & 1 & 7 & $\mathrm{H}(\mathrm{F}, \mathrm{X})$ \\
\hline Mycetophagidae & 1 & 1 & 1 & 1 & 1 & 5 & 1 & 7 & $\mathrm{~F}$ \\
\hline Nitidulidae & 0 & 1 & 1 & 1 & 0 & 1 & 1 & 2 & $\mathrm{~F}, \mathrm{~S}, \mathrm{H},(\mathrm{P})$ \\
\hline Ptilidae & 0 & 1 & 1 & 1 & 0 & 1 & 2 & 3 & $\mathrm{~F}$ \\
\hline Ptilodactylidae & 0 & 1 & 0 & 1 & 0 & 1 & 0 & 1 & $\mathrm{~F},(\mathrm{~S})$ \\
\hline Ptinidae & 0 & 5 & 2 & 6 & 0 & 7 & 2 & 9 & $\mathrm{~S}$ \\
\hline \multicolumn{10}{|l|}{ Scarabaeidae } \\
\hline Aphodiinae & 0 & 2 & 1 & 3 & 0 & 3 & 1 & 4 & S \\
\hline Cetoniinae & 0 & 1 & 0 & 1 & 0 & 12 & 0 & 12 & $\mathrm{H}(\mathrm{S}, \mathrm{X})$ \\
\hline Melolonthinae & 0 & 3 & 2 & 4 & 0 & 108 & 13 & 121 & $\mathrm{H}$ \\
\hline Scirtidae & 0 & 1 & 0 & 1 & 0 & 1 & 0 & 1 & S? \\
\hline \multicolumn{10}{|l|}{ Staphylinidae } \\
\hline Aleocharinae & 1 & 4 & 5 & 7 & 2 & 13 & 16 & 31 & $\mathrm{P}, \mathrm{F}, \mathrm{S}, \mathrm{H}$ \\
\hline Oxytelinae & 2 & 3 & 3 & 4 & 2 & 14 & 8 & 24 & $\mathrm{~S}, \mathrm{H}$ \\
\hline Paederinae & 0 & 1 & 0 & 1 & 0 & 2 & 0 & 2 & $\mathrm{P}$ \\
\hline Scydmaeninae & 0 & 0 & 1 & 1 & 0 & 0 & 1 & 1 & $\mathrm{P}$ \\
\hline Staphylininae & 0 & 3 & 0 & 3 & 0 & 3 & 0 & 3 & $\mathrm{P}$ \\
\hline Tachyporinae & 0 & 1 & 0 & 1 & 0 & 1 & 0 & 1 & $\mathrm{P}, \mathrm{S}, \mathrm{F}$ \\
\hline Tenebrionidae*** & 3 & 2 & 2 & 6 & 12 & 4 & 6 & 22 & $\mathrm{~S}, \mathrm{~F}$ \\
\hline Alleculinae & 0 & 3 & 2 & 4 & 0 & 6 & 4 & 10 & $\mathrm{~S}, \mathrm{~F}$ \\
\hline Total & 19 & 87 & 55 & 126 & $\mathbf{5 0}$ & 289 & 117 & 456 & \\
\hline
\end{tabular}


Cuadro 2. Número de especies raras (singletons y doubletons) y especies comunes (restantes) en tres diferentes estratos, representadas en número total y porcentaje del total de especies en cada estrato.

\begin{tabular}{ccccc}
\hline Estrato & $\mathbf{0 ~} \mathbf{~ m}$ & $\mathbf{1 ~} \mathbf{~}$ & $\mathbf{5} \mathbf{~ m}$ & $\begin{array}{c}\text { Total de la } \\
\text { muestra }\end{array}$ \\
\hline No. de especies & $\mathbf{1 9}$ & $\mathbf{8 7}$ & $\mathbf{5 5}$ & $\mathbf{1 2 6}$ \\
\hline Singletons & 10 & 54 & 37 & 73 \\
Doubletons & 5 & 15 & 3 & 18 \\
Restantes & 4 & 18 & 15 & 35 \\
\hline \% del total de especies & & & & \\
\hline Singletons & 52.6 & 62 & 67.3 & 58 \\
Doubletons & 26.3 & 17.2 & 5.4 & 14.3 \\
Restantes & 21 & 20.7 & 27.3 & 27.7 \\
\hline
\end{tabular}

Cuadro 3. Número de especies y familias exclusivas de la época de lluvias, sequía y presentes en ambos periodos, así como el número de individuos, en tres diferentes estratos, representadas en número total y porcentaje del total en cada estrato (en paréntesis).

\begin{tabular}{lcccc}
\hline Estrato & $\mathbf{0 ~ m}$ & $\mathbf{1} \mathbf{m}$ & $\mathbf{5} \mathbf{~ m}$ & $\begin{array}{c}\text { Total de la } \\
\text { muestra }\end{array}$ \\
\hline No. de especies & & & & \\
\hline Lluvias & $12(63.2 \%)$ & $60(69 \%)$ & $31(56.3 \%)$ & $78(62 \%)$ \\
Sequía & $4(21 \%)$ & $18(20.7 \%)$ & $14(25.4 \%)$ & $26(20.6 \%)$ \\
Lluvias-sequía & $3(15.8 \%)$ & $9(10.3 \%)$ & $10(18.2 \%)$ & $22(17.4 \%)$ \\
\hline No. de familias & & & & $14(45.2 \%)$ \\
\hline Lluvias & $7(58.3 \%)$ & $19(61.3 \%)$ & $10(43.5 \%)$ & $2(6.4 \%)$ \\
Sequía & $2(16.7 \%)$ & $1(3.2 \%)$ & $4(17.4 \%)$ & $15(48.4 \%)$ \\
Lluvias-sequía & $3(25 \%)$ & $11(35.5 \%)$ & $9(39.1 \%)$ & \\
\hline No. de individuos & & & & \\
\hline Lluvias & $37(74 \%)$ & $254(88 \%)$ & $82(70 \%)$ & $373(81.8 \%)$ \\
Sequía & $13(26 \%)$ & $35(12 \%)$ & $35(30 \%)$ & $83(18.2 \%)$ \\
\hline
\end{tabular}

Cuadro 4. Familias indicadoras para cada estrato con sus valores indicadores IndVal. El número de asteriscos indica el grado de significancia $\left(\mathrm{P}>0.01^{*}, \mathrm{P}>0.001 * *\right) .{ }^{1}$ Chrysomelidae sin Bruchinae.

\begin{tabular}{|c|c|c|c|}
\hline Estrato & Familias & IndVal & P. value \\
\hline \multirow[b]{2}{*}{$0 \mathrm{~m}$} & Carabidae & 0.327 & $0.0047^{* *}$ \\
\hline & Anthicidae & 0.326 & $0.0095 * *$ \\
\hline \multirow{6}{*}{$1 \mathrm{~m}$} & Coccinellidae & 0.365 & $0.0015 * *$ \\
\hline & Melolonthinae (Scarabaeidae) & 0.341 & $0.0026 * *$ \\
\hline & Mordellidae & 0.332 & $0.0062 * *$ \\
\hline & Ptinidae & 0.291 & $0.0136 *$ \\
\hline & Latridiidae & 0.275 & $0.0289 *$ \\
\hline & Chrysomelidae $^{1}$ & 0.269 & $0.0192 *$ \\
\hline \multirow[b]{2}{*}{$5 \mathrm{~m}$} & Bruchinae (Chrysomelidae) & 0.285 & $0.0222 *$ \\
\hline & Aleocharinae (Staphylinidae) & 0.255 & $0.0468 *$ \\
\hline
\end{tabular}


Cuadro 5. Lista de familias (representadas por $>10$ individuos) con el número de individuos (sobre la diagonal) y especies (debajo de la diagonal) capturados en tres estratos y tres sitios en un bosque artificial de México. * Chrysomelidae sin Bruchinae. **Curculionidae sin Scolytinae. $* * *$ Tenebrionidae sin Alleculinae.

\begin{tabular}{|c|c|c|c|c|c|c|c|c|c|c|}
\hline Estratos & $\mathbf{0} \mathbf{~ m}$ & & & $1 \mathrm{~m}$ & & & $5 \mathrm{~m}$ & & & \\
\hline Sitios & 1 & 2 & 3 & 1 & 2 & 3 & 1 & 2 & 3 & Total \\
\hline Cantharidae & $0 / 0$ & $1 / 1$ & $0 / 0$ & $8 / 2$ & $0 / 0$ & $2 / 1$ & $0 / 0$ & $0 / 0$ & $0 / 0$ & $11 / 4$ \\
\hline Carabidae & $5 / 2$ & $1 / 1$ & $6 / 1$ & $0 / 0$ & $0 / 0$ & $0 / 0$ & $2 / 2$ & $0 / 0$ & $4 / 3$ & $18 / 5$ \\
\hline Chrysomelidae* & $0 / 0$ & $0 / 0$ & $2 / 1$ & $7 / 3$ & $3 / 3$ & $4 / 3$ & $3 / 3$ & $1 / 1$ & $1 / 1$ & $21 / 12$ \\
\hline Bruchinae & $0 / 0$ & $0 / 0$ & $0 / 0$ & $2 / 2$ & $3 / 3$ & $4 / 3$ & $9 / 4$ & $1 / 1$ & $2 / 2$ & $21 / 7$ \\
\hline Curculionidae* & $0 / 0$ & $1 / 1$ & $1 / 1$ & $3 / 2$ & $0 / 0$ & $3 / 2$ & $0 / 0$ & $2 / 2$ & $4 / 3$ & $14 / 8$ \\
\hline Scolytinae & $0 / 0$ & $0 / 0$ & $0 / 0$ & $1 / 1$ & $1 / 1$ & $1 / 1$ & $1 / 1$ & $3 / 3$ & $3 / 3$ & $10 / 7$ \\
\hline Latridiidae & $1 / 1$ & $1 / 1$ & $2 / 1$ & $10 / 1$ & $2 / 1$ & $6 / 2$ & $3 / 1$ & $4 / 1$ & $3 / 1$ & $32 / 2$ \\
\hline Melandrydae & $0 / 0$ & $0 / 0$ & $0 / 0$ & $3 / 1$ & $1 / 1$ & $1 / 1$ & $3 / 1$ & $0 / 0$ & $2 / 1$ & $10 / 2$ \\
\hline Melyridae & $0 / 0$ & $1 / 1$ & $0 / 0$ & $1 / 1$ & $3 / 2$ & $4 / 1$ & $2 / 1$ & $1 / 1$ & $0 / 0$ & $12 / 2$ \\
\hline \multicolumn{11}{|l|}{ Scarabaeidae } \\
\hline Aphodiinae & $0 / 0$ & $0 / 0$ & $0 / 0$ & $0 / 0$ & $0 / 0$ & $3 / 2$ & $0 / 0$ & $0 / 0$ & $1 / 1$ & $4 / 3$ \\
\hline Cetoniinae & $0 / 0$ & $0 / 0$ & $0 / 0$ & $12 / 1$ & $0 / 0$ & $0 / 0$ & $0 / 0$ & $0 / 0$ & $0 / 0$ & $12 / 1$ \\
\hline Melolonthinae & $0 / 0$ & $0 / 0$ & $0 / 0$ & $59 / 2$ & $33 / 1$ & $16 / 2$ & $10 / 1$ & $1 / 1$ & $2 / 2$ & $121 / 4$ \\
\hline \multicolumn{11}{|l|}{ Staphylinidae } \\
\hline Aleocharinae & $0 / 0$ & $0 / 0$ & $2 / 1$ & $4 / 3$ & $4 / 2$ & $5 / 2$ & $6 / 4$ & $5 / 1$ & $5 / 2$ & $31 / 7$ \\
\hline Oxytelinae & $0 / 0$ & $0 / 0$ & $2 / 2$ & $2 / 2$ & $5 / 2$ & $7 / 3$ & $3 / 3$ & $2 / 2$ & $3 / 2$ & $24 / 4$ \\
\hline Paederinae & $0 / 0$ & $0 / 0$ & $0 / 0$ & $0 / 0$ & $0 / 0$ & $2 / 1$ & $0 / 0$ & $0 / 0$ & $0 / 0$ & $2 / 1$ \\
\hline Scydmaeninae & $0 / 0$ & $0 / 0$ & $0 / 0$ & $0 / 0$ & $0 / 0$ & $0 / 0$ & $0 / 0$ & $0 / 0$ & $1 / 1$ & $1 / 1$ \\
\hline Staphylininae & $0 / 0$ & $0 / 0$ & $0 / 0$ & $1 / 1$ & $2 / 2$ & $0 / 0$ & $0 / 0$ & $0 / 0$ & $0 / 0$ & $3 / 3$ \\
\hline Tachyporinae & $0 / 0$ & $0 / 0$ & $0 / 0$ & $0 / 0$ & $0 / 0$ & $1 / 1$ & $0 / 0$ & $0 / 0$ & $0 / 0$ & $1 / 1$ \\
\hline Tenebrionidae* & $0 / 0$ & $0 / 0$ & $12 / 3$ & $2 / 2$ & $1 / 1$ & $1 / 1$ & $4 / 2$ & $0 / 0$ & $2 / 1$ & $22 / 6$ \\
\hline Alleculinae & $0 / 0$ & $0 / 0$ & $0 / 0$ & $0 / 0$ & $4 / 2$ & $2 / 2$ & $0 / 0$ & $4 / 2$ & $0 / 0$ & $10 / 4$ \\
\hline Total & $6 / 3$ & $5 / 5$ & $27 / 10$ & $115 / 24$ & $62 / 21$ & $62 / 29$ & $46 / 23$ & $24 / 15$ & $33 / 23$ & $380 / 84$ \\
\hline
\end{tabular}

